\title{
(NON-)WEAKLY MIXING OPERATORS AND HYPERCYCLICITY SETS
}

\author{
F. BAYART AND É. MATHERON
}

\begin{abstract}
We study the frequency of hypercyclicity of hypercyclic, non-weakly mixing linear operators. In particular, we show that on the space $\ell^{1}(\mathbb{N})$, any sublinear frequency can be realized by a non-weakly mixing operator. A weaker but similar result is obtained for $c_{0}(\mathbb{N})$ or $\ell^{p}(\mathbb{N}), 1<p<\infty$. Part of our results is related to some Sidon-type lacunarity properties for sequences of natural numbers.
\end{abstract}

\section{INTRODUCTION}

The present paper is a contribution to the study of the dynamics of linear operators. More specifically, we are interested in the behaviour of individual orbits of a continuous linear operator. Most of the paper is devoted to the following question: how "frequently" can such an orbit visit each nonempty open set without forcing the operator to be topologically weakly mixing?

Let $X$ be a separable $F$-space over $\mathbb{K}=\mathbb{R}$ or $\mathbb{C}$, and let us denote by $\mathcal{L}(X)$ the set of all continuous linear operators on $X$. An operator $T \in \mathcal{L}(X)$ is said to be hypercyclic if there exists some vector $x \in X$ whose $T$-orbit $\left\{T^{n}(x) ; n \in \mathbb{N}\right\}$ is dense in $X$. Equivalently, $T$ is hypercyclic if and only if it is topologically transitive, which means that for any pair $\left(V, V^{\prime}\right)$ of nonempty open subsets of $X$, one can find some integer $n \in \mathbb{N}$ such that $T^{n}(V) \cap V^{\prime} \neq \varnothing$. An operator $T$ is said to be (topologically) weakly mixing if the operator $T \times T$ is hypercyclic on the product space $X \times X$.

Of course, hypercyclicity and weak mixing make sense for arbitrary continuous maps, and they are basic notions in topological dynamics. In the linear setting, weak mixing is particularly significant because it is closely related to the so-called Hypercyclicity Criterion. A linear operator $T \in \mathcal{L}(X)$ is said to satisfy the Hypercyclicity Criterion with respect to some increasing sequence of integers $\left(n_{k}\right)$ if one can find dense sets $D, D^{\prime} \subset X$ such that

(a) $T^{n_{k}}(x) \rightarrow 0$ for each $x \in D$;

(b) for each $x^{\prime} \in D^{\prime}$, one can find a sequence $\left(z_{k}\right) \subset X$ such that $z_{k} \rightarrow 0$ and $T^{n_{k}}\left(z_{k}\right) \rightarrow x^{\prime}$

2000 Mathematics Subject Classification. 47A16, 37B99, 11B99.

Key words and phrases. hypercyclic operators, weak mixing, Sidon sequences.

Aknowledgement: the authors would like to thank the anonymous referee for his/her careful reading, which led to the elimination of a number of mistakes. 
It is not difficult to show that operators satisfying the Hypercyclicity Criterion are indeed hypercyclic, and hence weakly mixing because $T \times T$ satisfies the criterion whenever $T$ does. By a nice result of J. Bès and A. Peris ([BeP $]$ ), the converse is also true: a linear operator satisfies the Hypercyclicity Criterion if and only if it is weakly mixing.

For many years, all known examples of hypercyclic operators were also known to satisfy the Hypercyclicity Criterion. Thus, it looked quite reasonable to conjecture that for linear operators, hypercyclicity and weak mixing are in fact equivalent. Nevertheless, M. De La Rosa and C. Read have shown very recently that hypercyclic operators need not be weakly mixing $([\mathrm{DR}])$. More precisely, they constructed a Banach space $X$ on which a counterexample exists. Building on their ideas, the authors of the present paper have proved that one can construct hypercyclic operators which are not weakly mixing on many classical Banach spaces (including all $\ell^{p_{-}}$ spaces), and also on the space of entire functions $\mathcal{H}(\mathbb{C})([\mathrm{BaM}])$.

On the other hand, several authors have observed that hypercyclicity combined with some qualitative property automatically yields weak mixing. One noteworthy example is a recent result of K. -G. Grosse-Erdmann and A. Peris ([GEP]), according to which every frequently hypercyclic operator is weakly mixing. Frequent hypercyclicity is defined as follows $([\mathrm{BaGr}])$. Let $T \in \mathcal{L}(X)$. For $x \in X$ and $V \subset X$ nonempty open, set

$$
\mathbf{N}(x, V):=\left\{n \in \mathbb{N} ; T^{n}(x) \in V\right\} .
$$

The operator $T$ is said to be frequently hypercyclic if there exists some vector $x \in X$ such that all sets $\mathbf{N}(x, V)$ have positive lower density; equivalently, if each set $\mathbf{N}(x, V)$ can be enumerated as an increasing sequence $\left(n_{k}\right)$ with $n_{k}=O(k)$ as $k \rightarrow \infty$.

In view of $[\mathrm{GEP}]$, it is not surprising that the operators constructed in $[\mathrm{DR}]$ and $[\mathrm{BaM}]$ seem very far from being frequently hypercyclic. The aim of the present paper is to try to fill the gap between frequently hypercyclic operators and these somewhat extreme examples, by investigating the frequency of hypercyclicity of non-weakly mixing operators.

Before formulating any result, we first have to give a precise meaning to the rather vague expression "frequency of hypercyclicity". The next definition is the most obvious generalization of frequent hypercyclicity.

Definition 1.1. Let $\left(m_{k}\right)_{k \in \mathbb{N}}$ be an increasing sequence of natural numbers. An operator $T \in \mathcal{L}(X)$ is said to be $\left(m_{k}\right)$-hypercyclic if there exists some vector $x \in X$ such that each set $\mathbf{N}(x, V)$ can be enumerated as an increasing sequence $\left(n_{k}\right)_{k \in \mathbb{N}}$ with $n_{k}=O\left(m_{k}\right)$ as $k \rightarrow \infty$.

Thus, the frequently hypercyclic operators are exactly the $(k)$-hypercyclic ones. The following theorem shows that, at least if one is allowed to choose the space $X$, the result of Grosse-Erdmann and Peris mentionned above is essentially optimal. 
Theorem 1.2. If $\left(m_{k}\right)$ is any increasing sequence of natural numbers such that $\lim _{k \rightarrow \infty} \frac{m_{k}}{k}=\infty$, then one can construct an operator on $X=\ell^{1}(\mathbb{N})$ which is $\left(m_{k}\right)$ hypercyclic, but not weakly mixing.

Corollary 1.3. For any $\varepsilon>0$, there exist non-weakly mixing operators on $\ell^{1}(\mathbb{N})$ which are $\left(k^{1+\varepsilon}\right)$-hypercyclic.

As stated, Theorem 1.2 just asserts the largeness of some sets of natural numbers $\mathbf{N}(x, V)$, without indicating any relation between these sets. However, if $x \in X$ is a hypercyclic vector for some operator $T \in \mathcal{L}(X)$, then the sets $\mathbf{N}(x, V)$ are in fact closely related to each other. More precisely, it is easily checked that if $V_{1}, V_{2}$ are two nonempty open subsets of $X$, then one can find a nonempty open set $\widetilde{V}_{1} \subset V_{1}$ and some natural number $N$ such that $N+\mathbf{N}\left(x, \widetilde{V}_{1}\right) \subset \mathbf{N}\left(x, V_{2}\right)$ : choose $N \in \mathbb{N}$ such that $T^{N}\left(V_{1}\right) \cap V_{2} \neq \varnothing$, and set $\widetilde{V}_{1}:=V_{1} \cap\left(T^{N}\right)^{-1}\left(V_{2}\right)$. Moreover, one can find an infinite set $\mathbf{B} \subset \mathbb{N}$ such that, for each neighbourhood $U$ of $x$, the set $\mathbf{N}(x, U)$ contains all but finitely many $n \in \mathbf{B}$ : just consider an increasing sequence $\left(b_{n}\right) \subset \mathbb{N}$ such that $T^{b_{n}}(x) \rightarrow x$ and set $\mathbf{B}:=\left\{b_{n} ; n \in \mathbb{N}\right\}$. From these two remarks, it follows in particular that each set $\mathbf{N}(x, V)$ contains a translate of some cofinite subset of $\mathbf{B}$. This suggests the following definition.

Definition 1.4. Let $T \in \mathcal{L}(X)$. An infinite set $\mathbf{B} \subset \mathbb{N}$ is said to be a hypercyclicity set for $T$ if there exists some vector $x \in X$ such that each set $\mathbf{N}(x, V)$ contains a translate of some cofinite subset of $\mathbf{B}$.

This definition is connected to the previous one in an obvious way: if $\mathbf{B}$ is an infinite subset of $\mathbb{N}$ whose increasing enumeration $\left(b_{n}\right)$ satisfies $b_{k+N}=O\left(m_{k}\right)$ as $k \rightarrow \infty$ for each fixed $N \in \mathbb{N}$, and if $\mathbf{B}$ is a hypercyclicity set for some operator $T$, then this operator is $\left(m_{k}\right)$-hypercyclic. And in fact, we will prove Theorem 1.2 by producing a set $\mathbf{B} \subset \mathbb{N}$ with the required growth property, to which one can associate some non-weakly mixing operator $T$ in such a way that $\mathbf{B}$ is a hypercyclicity set for $T$. Thus, we will have some vector $x \in X$ such that all sets $\mathbf{N}(x, V)$ are not merely large, but in fact large in exactly the same way.

It is not hard to convince oneself that the rate of growth of a hypercyclicity set cannot be arbitrary. In fact, we will prove below (Theorem 5.4) that an infinite set $\mathbf{B} \subset \mathbb{N}$ is a hypercyclicity set for some $F$-space operator $T$ if and only if its increasing enumeration $\left(b_{n}\right)$ satisfies $\lim _{n \rightarrow \infty}\left(b_{n+1}-b_{n}\right)=\infty$. But if one requires the operator $T$ to be non-weakly mixing, then the arithmetic properties of $\mathbf{B}$ come into play. This is apparent from [GEP], because what is actually proved there can be formulated as follows: If $T \in \mathcal{L}(X)$ and if there exists some vector $x \in X$ such that for each nonempty open set $V \subset X$, the difference set $\mathbf{N}(x, V)-\mathbf{N}(x, V)$ has bounded gaps (as it happens if $\mathbf{N}(x, V)$ has positive density), then $T$ is weakly mixing. For example, this rules out the possibility for the set $\mathbf{B}=\left\{n^{2} ; n \in \mathbb{N}\right\}$ to be a hypercyclicity set for any non-weakly mixing operator, even though its rate of growth is a priori admissible by 1.2 . 
Since arithmetical largeness of the sets $\mathbf{N}(x, V)$ entails weak mixing, it should not look very surprising that if, on the contrary, a set $\mathbf{B} \subset \mathbb{N}$ has some lacunarity property of arithmetical nature, then it turns out to be a hypercyclicity set for some non-weakly mixing operator. Recall that an increasing sequence of natural numbers $\left(b_{n}\right)_{n \in \mathbb{N}}$ is said to be a Sidon sequence if all sums $b_{k}+b_{l}$ with $k<l$ are distinct. We will need the following stronger Sidon-type property.

Definition 1.5. Let $\left(\Delta_{l}\right)_{l \in \mathbb{N}}$ be a sequence of natural numbers. An increasing sequence of natural numbers $\left(b_{n}\right)_{n \in \mathbb{N}}$ is said to be a $\left(\Delta_{l}\right)$-Sidon sequence if the sets of natural numbers $\mathbf{J}_{l}:=\bigcup_{k<l}\left[b_{l}+b_{k}, b_{l}+b_{k}+\Delta_{l}\right]$ are pairwise disjoint.

We now have the following result.

Theorem 1.6. Let $\left(b_{n}\right)_{n \in \mathbb{N}}$ be an increasing sequence of natural numbers. Assume that $\left(b_{n}\right)$ is $\left(\Delta_{l}\right)$-Sidon, for some sequence $\left(\Delta_{l}\right)$ tending to $\infty$. Then, for any infinite set $I \subset \mathbb{N}$, the set $\mathbf{B}:=\left\{b_{n} ; n \in \mathbb{N} \backslash I\right\}$ is a hypercyclicity set for some non-weakly mixing operator on $X=\ell^{1}(\mathbb{N})$.

Notice that one cannot hope to use this result to recover Theorem 1.2. Indeed, it is well known that the rate of growth of any Sidon sequence $\left(b_{n}\right)$ is at least $n^{2}$ (see [Hal]). On the other hand, Theorem 1.6 gives a large and natural class of subsets of $\mathbb{N}$ which are hypercyclicity sets for non-weakly mixing operators, while the set constructed in our proof of Theorem 1.2 seems to be a very peculiar one.

The two above theorems are stated for the space $X=\ell^{1}(\mathbb{N})$, and we are very far from being able to prove them on any separable Banach space. Yet, we do have some similar, though less satisfactory results on a rather large class of Banach spaces. Let us say that a linearly independent sequence $\left(e_{i}\right)_{i \in \mathbb{N}} \subset X$ is shift-admissible if the forward shift $S$ associated to $\left(e_{i}\right)$ is continuous. Recall that $S$ is the linear map on $\operatorname{span}\left\{e_{i} ; i \in \mathbb{N}\right\}$ defined by $S\left(e_{i}\right)=e_{i+1}$.

Theorem 1.7. Let $X$ be a separable Banach space, and assume $X$ has a normalized unconditional basis which is shift-admissible. Let also $\left(b_{n}\right)$ be an increasing sequence of natural numbers. Assume that $\left(b_{n}\right)$ is $\left(\Delta_{l}\right)$-Sidon, for some nondecreasing sequence $\left(\Delta_{l}\right)$ satisfying the following property: $\sum_{0}^{\infty} \exp \left(-\psi\left(\Delta_{l}\right)\right)<\infty$, where $\psi:(0, \infty) \rightarrow$ $(0, \infty)$ is a nondecreasing function such that $\int_{1}^{\infty} \frac{\psi(t)}{t^{2}} d t<\infty$. Then, for any infinite set $I \subset \mathbb{N}$, the set $\mathbf{B}:=\left\{b_{n} ; n \in \mathbb{N} \backslash I\right\}$ is a hypercyclicity set for some non-weakly mixing operator on $X$.

For any given $\varepsilon>0$, it is not hard to construct a sequence $\left(b_{n}\right)$ as above with $b_{k}=O\left(k^{3+\varepsilon}\right)$; see Lemma 3.2 below. Setting $I=2 \mathbb{N}$, we get the following corollary.

Corollary 1.8. Let the Banach space $X$ be as above. Then, for any $\varepsilon>0$ one can find an operator $T \in \mathcal{L}(X)$ which is $\left(k^{3+\varepsilon}\right)$-hypercyclic, but not weakly mixing.

Since, as we mentionned above, Sidon sequences cannot grow too slowly, it is very likely that this result does not give the optimal frequency of hypercyclicity for a 
non-weakly mixing operator on e.g. $c_{0}(\mathbb{N})$ or $\ell^{p}(\mathbb{N})$. It is quite plausible that one can still go down to $1+\varepsilon$, as in the $\ell^{1}$ case. However, we were not able to adapt our proof of Theorem 1.2 to this more general setting.

This paper is organized as follows. In Section 2, we describe our strategy for proving Theorems 1.2, 1.6 and 1.7. We introduce there the notations and give some preliminaries which will be useful throughout the whole paper. Section 3 is devoted to the proofs of Theorems 1.6 and 1.7. Theorem 1.2 is proved in section 4 . In Section 5, we give further informations on hypercyclicity sets. In particular, we give a criterion for an operator to admit a given set as a hypercyclicity set. In Section 6, we prove two results similar to 1.2 and 1.8 for a class of non-Banach Fréchet spaces. We conclude the paper by an expository section where we give unified proofs of several known results related to the Hypercyclicity Criterion.

\section{General framework}

2.1. The strategy. The following lemma from $[\mathrm{BaM}]$ isolates the basic idea used in $[\mathrm{DR}]$ and $[\mathrm{BaM}]$ to check that the operators considered there are not weakly mixing. Here and in the rest of the paper, we will use the following notations. If $T$ is a linear map defined on some vector space $Z$ and if $e_{0} \in Z$, we denote by $\mathbb{K}[T] e_{0}$ the linear span of the vectors $T^{i}\left(e_{0}\right), i \in \mathbb{N}$. In other words,

$$
\mathbb{K}[T] e_{0}=\left\{P(T) e_{0} ; P \text { polynomial }\right\} .
$$

Using the commutativity of the product of polynomials, it is easily checked that one can unambiguously define a product on $\mathbb{K}[T] e_{0}$ by the identities

$$
\left(P(T) e_{0}\right) \cdot\left(Q(T) e_{0}\right)=(P Q)(T) e_{0} .
$$

Lemma 2.1. Let $X$ be an $F$-space, and let $T \in \mathcal{L}(X)$ be hypercyclic with hypercyclic vector $e_{0}$. Assume there exists a nonzero linear functional $\phi: \mathbb{K}[T] e_{0} \rightarrow \mathbb{K}$ such that the map $(x, y) \mapsto \phi(x \cdot y)$ is continuous on $\mathbb{K}[T] e_{0} \times \mathbb{K}[T] e_{0}$. Then $T$ is not weakly mixing.

This lemma is essentially the same as Corollary 2.3 in [BaM]. Its proof is not difficult, but it seems unnecessary to give any detail at this point. We will say a few words on it in section 7 .

We can now describe our strategy for proving Theorems 1.2, 1.6 and 1.7. We start with a Banach space $X$ having a normalized, shift-admissible unconditional basis $\left(e_{i}\right)_{i \in \mathbb{N}}$, and we set

$$
c_{00}=\operatorname{span}\left\{e_{i} ; i \in \mathbb{N}\right\} .
$$

We denote by $\|\cdot\|_{1}$ the $\ell^{1}$-norm on $c_{00}$ : if $x=\sum_{i} x_{i} e_{i} \in c_{00}$, then $\|x\|_{1}=\sum_{i}\left|x_{i}\right|$.

We will define a linear map $T: c_{00} \rightarrow c_{00}$ depending on an increasing sequence of positive integers $\left(b_{n}\right)$, an infinite set $I$, and a number of additional parameters. By its very definition, the linear map $T$ will satisfy the following three properties. 
(i) $\operatorname{span}\left\{T^{i}\left(e_{0}\right) ; 0 \leq i \leq N\right\}=\operatorname{span}\left\{e_{i} ; 0 \leq i \leq N\right\}$ for all $N \in \mathbb{N}$, so that in particular $\mathbb{K}[T] e_{0}=c_{00}$.

(ii) Each vector $z=P(T) e_{0} \in \mathbb{K}[T] e_{0}$ is in the closure of the set $\left\{T^{b_{n}}\left(e_{0}\right) ; n \in I\right\}$.

(iii) $\lim _{\substack{n \rightarrow \infty \\ n \in \mathbb{N} \backslash I}} T^{b_{n}}\left(e_{0}\right)=e_{0}$.

It follows from (i) and (ii) that the set $\left\{T^{b_{n}}\left(e_{0}\right) ; n \in \mathbb{N}\right\}$ is dense in $X$. Hence, as soon as the linear map $T$ has been shown to be continuous, it extends to an operator on $X$ (still denoted by $T$ ) which is hypercyclic with hypercyclic vector $e_{0}$. Then property (iii) implies that the set $\mathbf{B}=\left\{b_{n} ; n \in \mathbb{N} \backslash I\right\}$ is a hypercyclicity set for $T$.

The strategy is now clear. To prove Theorems 1.6 and 1.7, we will show that, under the assumptions on $\left(b_{n}\right)$ stated therein and given the infinite set $I$, it is possible to choose the parameters involved in the definition of $T$ in such a way that $T$ is continuous, and one can define a linear functional $\phi$ satisfying the continuity property of Lemma 2.1. To prove Theorem 1.2, we will show that given the sequence $\left(m_{k}\right)$, one can choose the sequence $\left(b_{n}\right)$, the infinite set $I$ and the other parameters in such a way that $T$ is continuous, the linear functional $\phi$ can be constructed, and the increasing enumeration of any cofinite subset of $\mathbf{B}$ does not grow faster than the sequence $\left(m_{k}\right)$.

2.2. The parameters. The parameters involved in the construction of $T$ are the following:

- an increasing sequence of integers $\mathbf{b}=\left(b_{n}\right)_{n \in \mathbb{N}}$, with $b_{0}=0$;

- an infinite set $I \subset \mathbb{N}$, with $0 \in I$;

- a sequence of polynomials $\mathbf{P}=\left(P_{n}\right)_{n \in \mathbb{N}}$, with $P_{0}=0$;

- a nondecreasing sequence of positive numbers $\mathbf{a}=\left(a_{n}\right)$ tending to $\infty$, with $a_{0}=1$

- a nondecreasing sequence of positive numbers $(w(i))_{i \geq 1}$.

We will always assume that the following properties hold.

- $P_{n}=\mathbf{1}$ for all $n \in \mathbb{N} \backslash I$, and the sequence $\left(P_{n}\right)_{n \in I}$ enumerates (not in a 1-1 way) the set of all polynomials with coefficients in some fixed countable dense set $\mathbf{Q} \subset \mathbb{K}$.

- Setting $d_{n}:=\operatorname{deg}\left(P_{n}\right)$, we have $d_{n}<b_{n}$ for all $n$.

- The sequence $(w(i))$ has the form

$$
w(i)=K(1-\xi(i)),
$$

where $K \geq 4$ and $(\xi(i))_{i \geq 1}$ is a nonincreasing sequence of positive numbers tending to 0 , with $0<\xi(i) \leq \frac{1}{2}$ for all $i$. In particular, we have $2 \leq \frac{K}{2} \leq$ $w(i) \leq K$ for all $i$.

If $P$ is a polynomial, we denote by $|P|_{1}$ the sum of the moduli of its coefficients. 
2.3. The operator $T$. Since $\operatorname{deg}\left(P_{n}\right)<b_{n}$ for all $n$, there is a uniquely defined linear map $T: c_{00} \rightarrow c_{00}$ satisfying the following properties:

$$
\begin{cases}T\left(e_{i}\right)=w(i+1) e_{i+1} & \text { if } i \in \bigcup_{n \geq 1}\left[b_{n-1}, b_{n}-2\right] \\ T^{b_{n}}\left(e_{0}\right)=P_{n}(T) e_{0}+\frac{1}{a_{n}} e_{b_{n}} & \text { for all } n\end{cases}
$$

A simple computation shows that one can write

$$
T\left(e_{b_{n}-1}\right)=\varepsilon_{n} e_{b_{n}}+f_{n},
$$

where

$$
\varepsilon_{n}=\frac{a_{n-1}}{a_{n} w\left(b_{n-1}+1\right) \cdots w\left(b_{n}-1\right)}
$$

and

$$
f_{n}=\frac{a_{n-1}}{w\left(b_{n-1}+1\right) \cdots w\left(b_{n}-1\right)}\left(P_{n}(T) e_{0}-T^{b_{n}-b_{n-1}} P_{n-1}(T) e_{0}\right) .
$$

Since $d_{n}<b_{n}$ and $d_{n-1}<b_{n-1}$, the vector $f_{n}$ is supported on $\left[0, b_{n}\right)$. Thus, the operator $T$ satisfies property (i) stated above when describing our strategy. Moreover, (ii) and (iii) are also satisfied by definition of $T$, thanks to the assumptions made on the sequence $\left(P_{n}\right)$. The next lemma will be our main tool for checking the continuity of $T$. We set

$$
D_{n}:=b_{n}-b_{n-1} .
$$

Lemma 2.2. The following properties hold.

(a) $\varepsilon_{n} \leq 1$ for all $n \in \mathbb{N}^{*}$.

(b) If $n \in \mathbb{N}^{*}$ and if $\left\|f_{k}\right\|_{1} \leq 1$ for all $k<n$, then

$$
\begin{gathered}
\left\|f_{n}\right\|_{1} \leq C_{n}(K, \mathbf{a}, \mathbf{P})\left[(K / 2)^{-D_{n}}+\exp \left(-\sum_{i=1}^{D_{n}-1}\left(\xi\left(i+d_{n-1}\right)-\xi\left(i+b_{n-1}\right)\right)\right)\right], \\
\text { where } C_{n}(K, \mathbf{a}, \mathbf{P})=K^{\max \left(d_{n}, d_{n-1}\right)+1}\left|a_{n-1}\right|\left(\left|P_{n}\right|_{1}+\left|P_{n-1}\right|_{1}\right) .
\end{gathered}
$$

Proof. Part (a) is obvious. To prove (b), let us fix $n$ and assume $\left\|f_{k}\right\|_{1} \leq 1$ for all $k<n$. For each $j \in \mathbb{N}$, set $E_{j}:=\operatorname{span}\left(e_{0}, \ldots, e_{j}\right)$. Since the sequence $(w(i))$ is nondecreasing and $w(i) \geq 2$ for all $i$, it follows from our assumption that if $j<b_{n}$ and $x \in E_{j}$, one has $\|T x\|_{1} \leq w(j+1)\|x\|_{1}$. From this, we deduce that

$$
\left\|T^{j}\left(e_{0}\right)\right\|_{1} \leq \prod_{i=1}^{j} w(i)
$$

for all $j<b_{n}$. Looking at (3), this gives the inequality

$$
\left\|f_{n}\right\|_{1} \leq\left|a_{n-1}\right|\left|P_{n}\right|_{1} \frac{\prod_{i=1}^{d_{n}} w(i)}{\prod_{i=1}^{D_{n}-1} w\left(i+b_{n-1}\right)}+\left|a_{n-1}\right|\left|P_{n-1}\right|_{1} \frac{\prod_{i=1}^{D_{n}+d_{n-1}} w(i)}{\prod_{i=1}^{D_{n}-1} w\left(i+b_{n-1}\right)} .
$$


Since $K / 2 \leq w(i) \leq K$ for all $i$, the first term in the right-hand side of this inequality is not greater than

$$
\frac{K^{d_{n}}}{(K / 2)^{D_{n}-1}}\left|a_{n-1}\right|\left|P_{n}\right|_{1}
$$

and the second term does not exceed

$$
K^{d_{n-1}+1}\left|a_{n-1}\right|\left|P_{n-1}\right|_{1} \prod_{i=1}^{D_{n}-1} \frac{1-\xi\left(i+d_{n-1}\right)}{1-\xi\left(i+b_{n-1}\right)},
$$

as can be seen by writing the product in the numerator as $\prod_{i=1}^{D_{n}-1} w\left(i+d_{n-1}\right) \times$ $w\left(D_{n}+d_{n-1}\right) \times \prod_{i=1}^{d_{n-1}} w(i)$.

Taking the logarithm of the last product, using the inequality

$$
\log \left(\frac{1-v}{1-u}\right) \leq u-v
$$

$(0 \leq u \leq v<1)$ and adding the two estimates, we get (b).

2.4. The linear functional. Unlike the operator $T$, the linear functional $\phi$ we are looking for is not necessarily well-defined from the very beginning. What we need is a linear functional satisfying some special recurrence properties, which may very well be incompatible with each other. The precise definition is as follows.

Definition 2.3. Let $\boldsymbol{\Delta}=\left(\left(\Delta_{l}^{0}\right)_{l \in \mathbb{N}},\left(\Delta_{l}^{1}\right)_{l \in \mathbb{N}}\right)$ be a pair of sequences of natural numbers, with $\Delta_{0}^{i}=0$. For each $l \in \mathbb{N}$, set

$$
\mathbf{J}_{l}:=\left[b_{l}, b_{l}+\Delta_{l}^{0}\right] \cup \bigcup_{1 \leq k \leq l}\left[b_{l}+b_{k}, b_{l}+b_{k}+\Delta_{l}^{1}\right] .
$$

We will say that a linear functional $\phi: \mathbb{K}[T] e_{0} \rightarrow \mathbb{K}$ is $\Delta$-compatible if it has the following properties: $\phi\left(e_{0}\right)=1, \phi\left(T^{i} e_{0}\right)=0$ for all $i \in\left(0, b_{1}\right)$, and

$$
\phi\left(T^{i} e_{0}\right)= \begin{cases}\phi\left(P_{l}(T) T^{i-b_{l}} e_{0}\right) & \text { if } i \in \mathbf{J}_{l} \text { for some } l \geq 1 \\ 0 & \text { otherwise. }\end{cases}
$$

Notice that there is at most one $\boldsymbol{\Delta}$-compatible linear functional. This allows to speak of the $\boldsymbol{\Delta}$-compatible linear functional, even though it is perhaps not welldefined. The existence of this linear functional will depend on the properties of the sequence $\left(b_{n}\right)$. However, we can still list some of its properties assuming it is indeed well-defined. This is the content of the next lemma.

Lemma 2.4. Let $\boldsymbol{\Delta}=\left(\left(\Delta_{l}^{0}\right),\left(\Delta_{l}^{1}\right)\right)$ be a pair of sequences of natural numbers with $\Delta_{0}^{i}=0$, and assume the $\boldsymbol{\Delta}$-compatible linear functional $\phi$ is well-defined. For each $l \in \mathbb{N}$, set $\Delta_{l}=\min \left(\Delta_{l}^{0}, \Delta_{l}^{1}\right)$. Assume that $d_{k}:=\operatorname{deg}\left(P_{k}\right) \leq \Delta_{l} / 2$ whenever $0 \leq k \leq$ $l$, and that $\max \left(\Delta_{l}^{0}, \Delta_{l}^{1}\right)+2 d_{l}<\min \left(b_{l}, b_{l+1}-b_{l}\right)$ for all $l \geq 1$. Then the following holds for $\max (1, k) \leq l$ and $p=b_{k}+u \in\left[b_{k}, b_{k+1}\right), q=b_{l}+v \in\left[b_{l}, b_{l+1}\right)$. 
(a) $\phi\left(e_{p} \cdot e_{q}\right)=0$ if $u+v \leq \frac{\Delta_{l}}{2}$.

(b) $\left|\phi\left(e_{p} \cdot e_{q}\right)\right| \leq \frac{M_{l}(\mathbf{b}, \mathbf{a}, \mathbf{P})}{(K / 2)^{u+v}}$ if $u+v>\frac{\Delta_{l}}{2}$, where

$$
M_{l}(\mathbf{b}, \mathbf{a}, \mathbf{P})=4 a_{l}^{2} \prod_{j=1}^{2 b_{l+1}} \max \left(1,\left|P_{j}\right|_{1}\right)^{4} .
$$

Proof. By definition of $T$, we have

$$
e_{p} \cdot e_{q}=\frac{a_{k} a_{l}}{w\left(b_{k}+1\right) \cdots w\left(b_{k}+u\right) w\left(b_{l}+1\right) \cdots w\left(b_{l}+v\right)} y_{(k, u)(l, v)},
$$

where

$$
y_{(k, u)(l, v)}=\left(T^{b_{l}}-P_{l}(T)\right)\left(T^{b_{k}}-P_{k}(T)\right) T^{u+v} e_{0} .
$$

Assume $u+v \leq \frac{\Delta_{l}}{2}$. Then $z:=T^{b_{k}} T^{u+v} e_{0}$ is supported on $\left[b_{k}, b_{k}+\frac{\Delta_{l}}{2}\right] \subset \mathbf{J}_{l}-b_{l}$ and $z^{\prime}:=P_{k}(T) T^{u+v} e_{0}$ is supported on $\left[0, d_{k}+\frac{\Delta_{l}}{2}\right] \subset\left[0, \Delta_{l}\right] \subset \mathbf{J}_{l}-b_{l}$. By assumption on $\phi$, it follows that $\phi\left(\left(T^{b_{l}}-P_{l}(T)\right) z\right)=0=\phi\left(\left(T^{b_{l}}-P_{l}(T)\right) z^{\prime}\right)$, which gives (a).

We now turn to part (b). Notice that we have $\mathbf{J}_{0}=\{0\}$.

Claim 1. For any $l \geq 0$, we have $\sup _{i \in \mathbf{J}_{l}}\left|\phi\left(T^{i}\left(e_{0}\right)\right)\right| \leq \prod_{j=0}^{l} \max \left(1,\left|P_{j}\right|_{1}\right)^{2}$.

Proof of Claim 1. This is done by induction on $l$. The statement holds for $l=0$. Assume this has been proved for all $l^{\prime}<l$, and let $i \in \mathbf{J}_{l}$. It is easy to check that

$$
\left|\phi\left(T^{i}\left(e_{0}\right)\right)\right| \leq\left|P_{l}\right|_{1} \max _{m \leq i-b_{l}+d_{l}}\left|\phi\left(T^{m}\left(e_{0}\right)\right)\right| .
$$

Now, if $m \leq i-b_{l}+d_{l}$, then $m \leq b_{l}+\max \left(\Delta_{l}^{0}, \Delta_{l}^{1}\right)+d_{l}<b_{l+1}$ because $i \in \mathbf{J}_{l}$ and $\max \left(\Delta_{l}^{0}, \Delta_{l}^{1}\right)+d_{l}<b_{l+1}-b_{l}$. Hence, we have either $\phi\left(T^{m} e_{0}\right)=0$ or $m \in \mathbf{J}_{l^{\prime}}$ for some $l^{\prime} \leq l$. If $m \in \bigcup_{l^{\prime}<l} \mathbf{J}_{l^{\prime}}$, the induction hypothesis gives $\left|\phi\left(T^{m}\left(e_{0}\right)\right)\right| \leq$ $\prod_{j<l} \max \left(1,\left|P_{j}\right|_{1}\right)^{2}$. If $m \in \mathbf{J}_{l}$, then

$$
\begin{aligned}
\left|\phi\left(T^{m}\left(e_{0}\right)\right)\right| & \leq\left|P_{l}\right|_{1} \max _{j \leq m-b_{l}+d_{l}}\left|\phi\left(T^{j}\left(e_{0}\right)\right)\right| \\
& \leq\left|P_{l}\right|_{1} \prod_{j<l} \max \left(1,\left|P_{j}\right|_{1}\right)^{2},
\end{aligned}
$$

where the second inequality follows from the induction hypothesis, since $m-b_{l}+d_{l} \leq$ $i-2 b_{l}+2 d_{l} \leq \max \left(\Delta_{l}^{0}, \Delta_{l}^{1}\right)+2 d_{l}<b_{l}$. In either case, we get the required estimate for $\left|\phi\left(T^{i}\left(e_{0}\right)\right)\right|$.

Claim 2. We have $\left|\phi\left(y_{(k, u)(l, v)}\right)\right| \leq 4 \prod_{j=0}^{2 b_{l}+1} \max \left(1,\left|P_{j}\right|_{1}\right)^{4}$. 
Proof of Claim 2. This follows directly from Claim 1 using the inequalities

$$
\begin{aligned}
\left|\left(t^{b_{k}}-P_{k}\right)\left(t^{b_{l}}-P_{l}\right)\right|_{1} & \leq\left(1+\left|P_{k}\right|_{1}\right)\left(1+\left|P_{l}\right|_{1}\right) \\
& \leq 4 \max \left(1,\left|P_{k}\right|_{1}\right) \max \left(1,\left|P_{l}\right|_{1}\right)
\end{aligned}
$$

and

$$
b_{k}+b_{l}+u+v \leq 2 b_{l+1} \leq b_{2 b_{l+1}} .
$$

The estimate (b) in 2.4 is now an immediate consequence of Claim 2.

Remark. The above proof also gives the following estimate, which will be useful in Section 6. Set $i(l):=\min \left\{i ; b_{i+1}-b_{i}>\frac{\Delta_{l}}{4}\right\}$. If $u+v>\frac{\Delta_{l}}{2}$, then

$$
\left|\phi\left(e_{p} \cdot e_{q}\right)\right| \leq \frac{M_{l}(\mathbf{b}, \mathbf{a}, \mathbf{P})}{w\left(b_{i(l)}\right)^{\Delta_{l} / 4}} .
$$

Indeed, one has either $u>\frac{\Delta_{l}}{4}$ or $v>\frac{\Delta_{l}}{4}$, whereas $u<b_{k+1}-b_{k}$ and $v<b_{l+1}-b_{l}$.

\section{Strong Sidon sequences}

3.1. What we have to do. In this section, we prove Theorems 1.6 and 1.7. So we start with a sequence $\left(b_{n}\right)$ which is $\left(\Delta_{l}\right)$-Sidon and with an infinite set $I \subset \mathbb{N}$. Without loss of generality, we may assume that $b_{0}=0=\Delta_{0}$ and $0 \in I$.

Having fixed $\left(b_{n}\right)$ and $I$, we have at hand a linear map $T: c_{00} \rightarrow c_{00}$ depending on several parameters. Our goal is to show that, in each of the two considered cases, one can choose these parameters in such a way that $T$ is continuous, and a linear functional $\phi: \mathbb{K}[T] e_{0} \rightarrow \mathbb{K}$ with the required continuity property can be constructed.

By assumption, the sets $\mathbf{J}_{l}=\bigcup_{k \leq l}\left[b_{l}+b_{k}, b_{l}+b_{k}+\Delta_{l}\right]$ are pairwise disjoint. Setting $\boldsymbol{\Delta}:=\left(\left(\Delta_{l}\right),\left(\Delta_{l}\right)\right)$, it follows that, whatever the choice of the parameters may be, the $\Delta$-compatible linear functional $\phi: \mathbb{K}[T] e_{0} \rightarrow \mathbb{K}$ is well-defined. Thus, it remains to show that if the parameters are suitably chosen, then $T$ is continuous and the map $(x, y) \mapsto \phi(x \cdot y)$ is continuous on $\mathbb{K}[T] e_{0} \times \mathbb{K}[T] e_{0}$. With the notations of Lemmas 2.2 and 2.4 , it is enough to ensure:

- in the $\ell^{1}$-case, that the sequence $\left(\left\|f_{n}\right\|_{1}\right)_{n \geq 1}$ is bounded as well as the double sequence $\left(\phi\left(e_{p} \cdot e_{q}\right)\right)_{p, q \in \mathbb{N}}$;

- in the "general" case, that $\sum_{1}^{\infty}\left\|f_{n}\right\|_{1}<\infty$ and $\sum_{p, q}\left|\phi\left(e_{p} \cdot e_{q}\right)\right|<\infty$.

Indeed, the operator $T$ has the form

$$
T=R+L,
$$


where $R$ is a forward shift with bounded weights, and $L$ is defined on $c_{00}$ by

$$
L\left(\sum_{i} x_{i} e_{i}\right)=\sum_{n} x_{b_{n}-1} f_{n} .
$$

Since the basis $\left(e_{i}\right)$ is shift-admissible and unconditional, the operator $R$ is continuous. And since $\left(e_{i}\right)$ is normalized, it is readily seen that the operator $L$ is continuous if the appropriate condition on $\left(f_{n}\right)$ is satisfied. Likewise, the bilinear map $(x, y) \mapsto \phi(x \cdot y)$ is continuous if the corresponding condition on $\left(\phi\left(e_{p} \cdot e_{q}\right)\right)$ is fulfilled.

3.2. Proof of Theorem 1.6. We first isolate a lemma ensuring the continuity of $T$ on $\ell^{1}(\mathbb{N})$. This lemma will also be needed in section 4 .

Lemma 3.1. Assume that $b_{n}-b_{n-1} \rightarrow \infty$, and that $\lim _{i \rightarrow \infty} i[\xi(2 i)-\xi(3 i)]=\infty$. Then one can find a sequence $\left(u_{n}\right)$ tending to $\infty$ such that the following holds: whenever the sequences $\left(P_{n}\right)$ and $\left(a_{n}\right)$ are chosen in such a way that $a_{n} \leq u_{n}, d_{n} \leq u_{n}$ and $\left|P_{n}\right|_{1} \leq u_{n}$ for all $n \in \mathbb{N}$, it follows that $T$ is continuous on $\ell^{1}(\mathbb{N})$.

Proof. We first choose another sequence $\left(\widetilde{D}_{n}\right)$ tending to infinity and such that $\widetilde{D}_{n}<$ $\min \left(\frac{b_{n-1}}{3}, b_{n}-b_{n-1}\right)$ for all $n \geq 1$.

Assume that the sequence of polynomials $\left(P_{n}\right)$ satisfies $d_{n-1}<\widetilde{D}_{n}$ for all $n \geq 1$. With the notations of Lemma 2.2 and since $D_{n}:=b_{n}-b_{n-1}>\widetilde{D}_{n}$, we have

$$
\begin{aligned}
\sum_{i=1}^{D_{n}-1}\left(\xi\left(i+d_{n-1}\right)-\xi\left(i+b_{n-1}\right)\right) & \geq \sum_{i=1}^{\widetilde{D}_{n}}\left(\xi\left(i+d_{n-1}\right)-\xi\left(i+b_{n-1}\right)\right) \\
& \geq \widetilde{D}_{n} \times\left(\xi\left(2 \widetilde{D}_{n}\right)-\xi\left(3 \widetilde{D}_{n}\right)\right) .
\end{aligned}
$$

By assumption on $\xi$, it follows that

$$
\lim _{n \rightarrow \infty} \sum_{i=1}^{D_{n}-1}\left(\xi\left(i+d_{n-1}\right)-\xi\left(i+b_{n-1}\right)\right)=\infty .
$$

By Lemma 2.2, it is now clear that since $K>2$, one can find a sequence $\left(u_{n}\right)$ tending to $\infty$ such that the following holds: whenever the sequences $\left(P_{n}\right)$ and $\left(a_{n}\right)$ are chosen in such a way that $a_{n} \leq u_{n}, d_{n} \leq u_{n}$ and $\left|P_{n}\right|_{1} \leq u_{n}$ for all $n \in \mathbb{N}$, it follows that $\left\|f_{n}\right\|_{1} \leq 1$ for any $n$.

Remark. The conclusion of Lemma 3.1 does not hold if $b_{n}-b_{n-1}$ does not go to $\infty$. Indeed, if $b_{n}-b_{n-1} \leq C$ along some increasing sequence $\left(n_{k}\right)$ and if we choose $\left(P_{n}\right)$ in such a way that $P_{n_{k}}=\mathbf{1}$ and $P_{n_{k}-1}=0$ for all $k$, then we get $\left\|f_{n_{k}}\right\|_{1} \geq \frac{a_{n_{k}-1}}{K^{C}-1}$, whence $\lim \sup \left\|f_{n}\right\|=\infty$.

Proof of Theorem 1.6. First observe that since $\left(b_{n}\right)$ is $\left(\Delta_{l}\right)$-Sidon, we have $b_{n}-b_{n-1}>$ $\Delta_{n-1}$ for all $n \geq 1$, so that $b_{n}-b_{n-1}$ goes to infinity. Thus, we may call on the previous 
lemma to get that $T$ is continuous, provided $\left(a_{n}\right)$ and $\left(P_{n}\right)$ are suitably controlled by some sequence $\left(u_{n}\right)$ and e.g. $\xi(i)=\frac{1}{2 \sqrt{i}}$.

With the notations of Lemma 2.4 and since $\Delta_{l} \rightarrow \infty$, one can choose the sequences $\left(a_{n}\right)$ and $\left(P_{n}\right)$ in such a way that $\operatorname{deg}\left(P_{k}\right) \leq \Delta_{l}$ whenever $k \leq l$ and $M_{l}(\mathbf{a}, \mathbf{b}, \mathbf{P}) \leq$ $2^{\Delta_{l} / 2}$ for all $l \geq 1$; and of course, this choice can be made consistently with the above control by the sequence $\left(u_{n}\right)$. Since $K \geq 4$, it follows that the double sequence $\left(\phi\left(e_{p} \cdot e_{q}\right)\right)$ is bounded. This concludes the proof of 1.6.

3.3. Proof of Theorem 1.7. Since $\left(b_{n}\right)$ is $\left(\Delta_{l}\right)$-Sidon, we have $D_{n}:=b_{n}-b_{n-1}>$ $\Delta_{n-1}$ for all $n \geq 1$. Moreover, replacing $\Delta_{l}$ by $\tilde{\Delta}_{l}:=\min \left(\Delta_{l}, \frac{\left[b_{l}\right]}{3}\right)$ and $\psi(t)$ by $\tilde{\psi}(t):=\max (\psi(t), \sqrt{t})$ if necessary, we may also assume that $\Delta_{n-1} \leq \frac{b_{n-1}}{3}$ for all $n \geq 1$. Then, exactly as in the proof of Theorem 1.6, we get the estimate

$$
\sum_{i=1}^{D_{n}-1}\left(\xi\left(i+d_{n-1}\right)-\xi\left(i+b_{n-1}\right)\right) \geq \Delta_{n-1} \times\left(\xi\left(2 \Delta_{n-1}\right)-\xi\left(3 \Delta_{n-1}\right)\right) .
$$

Now, we choose a continuous decreasing function $\xi:(0, \infty) \rightarrow(0, \infty)$ such that $0<\xi(t) \leq \frac{1}{2}$ for all $t$ and

$$
\xi(t)=6 \int_{t}^{\infty} \frac{\psi(s / 2)}{s^{2}} d s
$$

for large enough $t$. Since $\psi$ is nondecreasing, we have

$$
\begin{aligned}
\xi\left(2 \Delta_{n-1}\right)-\xi\left(3 \Delta_{n-1}\right) & =6 \int_{2 \Delta_{n-1}}^{3 \Delta_{n-1}} \frac{\psi(s / 2)}{s^{2}} d s \\
& \geq 6 \psi\left(\Delta_{n-1}\right) \times\left(\frac{1}{2 \Delta_{n-1}}-\frac{1}{3 \Delta_{n-1}}\right)
\end{aligned}
$$

for large enough $n$, hence

$$
\sum_{i=1}^{D_{n}-1}\left(\xi\left(i+d_{n-1}\right)-\xi\left(i+b_{n-1}\right)\right) \geq \psi\left(\Delta_{n-1}\right) .
$$

Moreover, we have $\int_{t}^{\infty} \frac{\psi(s)}{s^{2}} d s \geq \frac{\psi(t)}{t}$ for all $t \geq 1$, so that $\psi(t)=o(t)$ as $t \rightarrow \infty$. Consequently, we have $\sum_{0}^{\infty} c^{\Delta_{n}}<\infty$ for any $c \in(0,1)$, and hence $\sum_{1}^{\infty} c^{D_{n}}<\infty$. By Lemma 2.2 and since $K>2$, it follows that one can find some sequence $\left(u_{n}\right)$ tending to $\infty$ such that $\sum_{1}^{\infty}\left\|f_{n}\right\|_{1}<\infty$ whenever the sequences $\left(a_{n}\right)$ and $\left(P_{n}\right)$ are controlled by $\left(u_{n}\right)$ as in Lemma 3.1.

Finally, we show that $\phi$ has the required continuity property if the parameters are suitably chosen. Since $\Delta_{l}$ clearly goes to infinity, one can assume that $\operatorname{deg}\left(P_{k}\right) \leq \Delta_{l} / 2$ whenever $k \leq l$, consistently with the control by the sequence $\left(u_{n}\right)$. Applying Lemma 
2.4 , we get

$$
\begin{aligned}
\sum_{p, q}\left|\phi\left(e_{p} \cdot e_{q}\right)\right| & \leq 2 \sum_{k \geq 0} \sum_{l \geq k} M_{l}(\mathbf{a}, \mathbf{b}, \mathbf{P}) \sum_{u+v>\frac{\Delta_{l}}{2}}\left(\frac{K}{2}\right)^{-(u+v)} \\
& \leq 2 \sum_{k \geq 0} \sum_{l \geq k} M_{l}(\mathbf{a}, \mathbf{b}, \mathbf{P}) \sum_{j>\frac{\Delta_{l}}{2}}(j+1) \times\left(\frac{K}{2}\right)^{-j} \\
& \leq C \sum_{k \geq 0} \sum_{l \geq k} M_{l}(\mathbf{a}, \mathbf{b}, \mathbf{P})\left(\frac{K}{4}\right)^{-\Delta_{l} / 2}
\end{aligned}
$$

for some absolute constant $C<\infty$. Hence, we get

$$
\sum_{p, q}\left|\phi\left(e_{p} \cdot e_{q}\right)\right| \leq\left(\sum_{k \geq 0}\left(\frac{K}{4}\right)^{-\Delta_{k} / 4}\right) \times\left(\sum_{l \geq 0} M_{l}(\mathbf{a}, \mathbf{b}, \mathbf{P})\left(\frac{K}{4}\right)^{-\Delta_{l} / 4}\right),
$$

where we have used the fact that the sequence $\left(\Delta_{l}\right)$ is nondecreasing. Now, fix $c \in(0,1)$. We observed above that $\sum_{0}^{\infty} c^{\Delta_{n}}<\infty$, so one can always choose the sequences $\left(P_{n}\right)$ and $\left(a_{n}\right)$ in such a way that $\sum_{l \geq 0} M_{l}(\mathbf{a}, \mathbf{b}, \mathbf{P}) c^{\Delta_{l}}<\infty$, consistently with the control by the sequence $\left(u_{n}\right)$. It follows that if $K>4 c^{-4}$, then one can ensure $\sum_{p, q}\left|\phi\left(e_{p} \cdot e_{q}\right)\right|<\infty$.

3.4. The greedy algorithm. If we want Theorems 1.6 and 1.7 to be meaningful, we certainly have to exhibit some $\left(\Delta_{l}\right)$-Sidon sequences. We use a variant of the naive greedy algorithm for Sidon sequences (see [Hal]), which yields immediately Corollary 1.8 .

Lemma 3.2. Let $\varepsilon>0$ be given. If $0<\alpha<\frac{\varepsilon}{2}$, then one can find an increasing sequence of natural numbers $\left(b_{n}\right)_{n \in \mathbb{N}}$ with $b_{0}=0$, which is $\left(\left[l^{\alpha}\right]\right)$-Sidon and such that $b_{n}=O\left(n^{3+\varepsilon}\right)$.

Proof. Let $b_{0}=0$. We build the sequence $\left(b_{n}\right)$ by setting for $n \geq 1$ :

$$
\begin{aligned}
A_{n} & :=\left\{b_{k}+b_{l}+u-\left(b_{m}+v\right) ; k \leq l \leq n-1, u \leq\left[l^{\alpha}\right], m \leq n-1, v \leq\left[n^{\alpha}\right]\right\}, \\
B_{n} & :=\left\{b_{k}+b_{l}+u-v ; k \leq l \leq n-1, u \leq\left[l^{\alpha}\right], v \leq\left[n^{\alpha}\right]\right\}, \text { and } \\
b_{n} & :=\min \left(\mathbb{N} \backslash\left(A_{n} \cup \frac{1}{2} B_{n}\right)\right) .
\end{aligned}
$$

By its very definition, the sequence $\left(b_{n}\right)$ is increasing and $\left(\left[l^{\alpha}\right]\right)$-Sidon. Indeed, suppose that an equality $b_{n}+b_{m}+v=b_{l}+b_{k}+u$ holds, with $m \leq n, k \leq l \leq n-1$ and $u \leq\left[l^{\alpha}\right], v \leq\left[n^{\alpha}\right]$. Then if $m<n$, this equality is impossible because $b_{n} \notin A_{n}$; and if $m=n$, this is also impossible because $2 b_{n} \notin B_{n}$.

Moreover, since $\sharp A_{n} \leq n^{3} \times\left(1+\left[n^{\alpha}\right]\right)^{2}$ and $\sharp B_{n} \leq n^{2} \times\left(1+\left[n^{\alpha}\right]\right)^{2}$ for all $n$, we have $b_{n}=O\left(n^{3+\varepsilon}\right)$. 
Remark 1. The best known admissible upper bound for the growth of a Sidon sequence is $O\left(n^{\frac{1}{\sqrt{2}-1}+\varepsilon}\right)$ for any $\varepsilon>0$; this is due to I. Rusza [Rus]. One could try to adapt his method for getting a better bound for $\left(\Delta_{l}\right)$-Sidon sequences, but as we already mentionned, this approach would most likely not give the best frequency of hypercyclicity on e.g. $c_{0}(\mathbb{N})$ or $\ell^{p}(\mathbb{N})$.

Remark 2. In addition, one can also require that $b_{n} \geq n^{3+\varepsilon}$ for all $n$ : just set $C_{n}:=$ $\left[0, n^{3+\varepsilon}\right)$ and $b_{n}=\min \left(\mathbb{N} \backslash\left(A_{n} \cup \frac{1}{2} B_{n} \cup C_{n}\right)\right)$. This will be needed in section 6 .

3.5. Comparison with the operator defined in $[\mathrm{BaM}]$. The operator $T$ has formally the same definition as the one introduced in $[\mathrm{BaM}]$, except that in the present paper we allow the weights $w(i)$ to vary, while in $[\mathrm{BaM}]$ we had $w(i)=2$ for all $i$. However, there is an important difference in the proof of continuity. In [BaM], the sequences $\left(P_{n}\right)$ and $\left(a_{n}\right)$ were fixed, and we were able to prove the continuity of $T$ provided $\left(b_{n}\right)$ was sufficiently fast increasing. Here, the rule of the game is that the sequence $\left(b_{n}\right)$ is given a priori. Then we try to choose $\left(P_{n}\right)$ and $\left(a_{n}\right)$ in such a way that $T$ is continuous. In spite of this, the proof of continuity given in the present paper (in particular that of Lemma 3.1) is rather less technical than the corresponding one in $[\mathrm{BaM}]$, because one can take advantage of the freedom allowed on the weights $w(i)$.

\section{The optimal Frequency of HyperCyClicity on $\ell^{1}(\mathbb{N})$}

4.1. The sequence $\left(b_{n}\right)$. The aim of this section is to prove Theorem 1.2. So we fix an increasing sequence of natural numbers $\left(m_{k}\right)$ such that $\lim _{k \rightarrow \infty} \frac{m_{k}}{k}=\infty$. We will first define the increasing sequence $\left(b_{n}\right)$ and the infinite set $I \subset \mathbb{N}$, and then show that the other parameters involved in the definition of $T$ can be suitably chosen.

Lemma 4.1. Let $\left(q_{p}\right)_{p \in \mathbb{N}}$ be an increasing sequence of natural numbers with $q_{0}=0$, and set $I:=\left\{q_{p} ; p \in \mathbb{N}\right\}$. If the sequence $\left(q_{p}\right)_{p \geq 1}$ is sufficiently fast increasing, then one can construct an increasing sequence of natural numbers $\left(b_{n}\right)_{n \in \mathbb{N}}$ such that:

(1) $b_{0}=0=q_{0}$;

(2) $b_{n+1} \geq 4 b_{n}$ if $n=q_{p}-1$ or $q_{p}$ for some $p \geq 1$;

(3) the following congruences modulo 3 hold:

$$
\begin{cases}b_{n} \equiv 0(\bmod 3) & \text { for all } n \in \mathbb{N} \backslash I \\ b_{q_{p}} \equiv 1(\bmod 3) & \text { for all } p \geq 1\end{cases}
$$

(4) for each $p$, the following congruences modulo $2^{p+1}$ hold:

$$
\begin{cases}b_{q_{p}} \equiv 2^{p}\left(\bmod 2^{p+1}\right) & \text { if } p \geq 1 \\ b_{n} \equiv 0\left(\bmod 2^{p+1}\right) & \text { whenever } n>q_{p} \geq 0\end{cases}
$$

(5) $b_{2 k}=O\left(m_{k}\right)$. 
Proof. By the Chinese Remainder Theorem, one can construct inductively a sequence $\left(b_{n}\right)$ satisfying properties $(1), \ldots,(4)$ above and the following additional requirements: $b_{q_{p}} \leq 4 b_{q_{p}-1}+C_{p}$ for all $p \geq 1$, and $b_{q_{p}+j} \leq 4 b_{q_{p}}+C_{p} j$ if $p \geq 0$ and $1 \leq j<q_{p+1}-q_{p}-1$, where $C_{p}=3 \times 2^{p+1}$. Then $b_{k} \leq A_{p(k)}+B_{p(k)} k$ for all $k \in \mathbb{N}$, where the sequences $\left(A_{p}\right),\left(B_{p}\right)$ are independent of $\left(q_{p}\right)$ and $\left(b_{n}\right)$, and $p(k)=\max \left\{p ; q_{p} \leq k\right\}$. Since $\frac{m_{k}}{k} \rightarrow \infty$, we conclude that (5) is satisfied if the sequence $\left(q_{p}\right)$ is sufficiently fast increasing.

4.2. The $\Delta$-compatible linear functional. From now on, we fix a fast increasing sequence of natural numbers $\left(q_{p}\right)_{p \in \mathbb{N}}$ with $q_{0}=0$, and a sequence $\left(b_{n}\right)$ satisfying properties $(1), \ldots,(5)$ stated in Lemma 4.1. As in 4.1 , we set $I=\left\{q_{p} ; p \in \mathbb{N}\right\}$. At this point, the situation is rather more complicated than for Theorems 1.6 and 1.7: since $\left(b_{n}\right)$ is not at all a Sidon sequence, there is a priori no reason why any $\Delta$-compatible linear functional should be well-defined. But since $P_{n}$ is often equal to 1 and because the $b_{q_{p}}$ 's are well identified and separated in the sequence $\left(b_{n}\right)$, one can overcome this difficulty. This is the content of the next lemma.

Lemma 4.2. One can find a pair $\boldsymbol{\Delta}$ of nondecreasing sequences of natural numbers tending to $\infty$ such that the $\boldsymbol{\Delta}$-compatible linear functional $\phi$ is well-defined.

Proof. Let $(\alpha(r))_{r \in \mathbb{N}}$ and $(\beta(r))_{r \in \mathbb{N}}$ be two increasing sequences of natural numbers satisfying the following properties:

- $\alpha(0)=0=\beta(0)$;

- $\beta(r-1) \leq \alpha(r) \leq \beta(r)$ for all $r \geq 1$;

- $\beta(r) \geq b_{q_{\alpha(r)}}:=\theta(r)$ and $2^{\beta(r)}>r+b_{q_{\theta(r)}}$ for all $r$.

We define the two sequences $\left(\Delta_{l}^{0}\right)$ and $\left(\Delta_{l}^{1}\right)$ as follows:

$$
\begin{cases}\Delta_{l}^{0}=r & \text { if } \quad l \in\left[q_{p}, q_{p+1}\right) \text { and } p \in[\alpha(r), \alpha(r+1)) \\ \Delta_{l}^{1}=r & \text { if } \quad l \in\left[q_{p}, q_{p+1}\right) \text { and } p \in[\beta(r), \beta(r+1))\end{cases}
$$

Observe that $\Delta_{l}^{1} \leq \Delta_{l}^{0}$ (since $\beta(r) \geq \alpha(r)$ ) and $\Delta_{l}^{0} \leq b_{l}$ because the sequences $\left(b_{n}\right),\left(q_{j}\right),(\alpha(r))$ and $(\beta(r))$ are increasing.

With the usual notation

$$
\mathbf{J}_{l}:=\left[b_{l}, b_{l}+\Delta_{l}^{0}\right] \cup \bigcup_{1 \leq k \leq l}\left[b_{l}+b_{k}, b_{l}+b_{k}+\Delta_{l}^{1}\right],
$$

we have to show that it is possible to define $\phi\left(T^{i}\left(e_{0}\right)\right)$ for all $i \in \mathbb{N}$ in such a way that $\phi\left(e_{0}\right)=1, \phi\left(T^{i}\left(e_{0}\right)\right)=0$ for $i \in\left(0, b_{1}\right)$ and

$$
\phi\left(T^{i}\left(e_{0}\right)\right)=\phi\left(P_{l}(T) T^{i-b_{l}} e_{0}\right)
$$

whenever $i \in \mathbf{J}_{l}$ for some $l \geq 1$.

We prove that by induction on $i$. There is nothing to do if $i<b_{1}$, so let us fix $i \geq b_{1}$, and assume $\phi\left(T^{j}\left(e_{0}\right)\right)$ has been consistently defined for all $j<i$. We have 
to show that if $i$ belongs to several sets $\mathbf{J}_{l}$, then the possible definitions of $\phi\left(T^{i}\left(e_{0}\right)\right)$ coincide. In other words, we need to check that if $1 \leq l<l^{\prime}$ and $i$ can be written as

$$
b_{l}+b_{k}+u=i=b_{l^{\prime}}+b_{k^{\prime}}+v,
$$

where $k \leq l, k^{\prime} \leq l^{\prime}$ and $u \leq \Delta_{l}^{\min (k, 1)}, v \leq \Delta_{l^{\prime}}^{\min \left(k^{\prime}, 1\right)}$, then the "compatibility equation"

$$
\phi\left(P_{l}(T) T^{b_{k}+u} e_{0}\right)=\phi\left(P_{l^{\prime}}(T) T^{b_{k^{\prime}}+v} e_{0}\right)
$$

is satisfied (the two terms of this equation are well-defined since $\operatorname{deg}\left(P_{l}\right)<b_{l}$ and $\left.\operatorname{deg}\left(P_{l^{\prime}}\right)<b_{l^{\prime}}\right)$. Let us fix two such triples $(l, k, u)$ and $\left(l^{\prime}, k^{\prime}, v\right)$.

Fact 1. There exists some integer $p \in \mathbb{N}$ such that $l, l^{\prime} \in\left(q_{p}, q_{p+1}\right)$. In particular, both $l$ and $l^{\prime}$ belong to $\mathbb{N} \backslash I$.

Proof of Fact 1. Otherwise, one can find an integer $m \geq 1$ such that either $l \leq$ $q_{m}<l^{\prime}$ or $l<q_{m} \leq l^{\prime}$. In any case, property (2) of Lemma 4.1 gives $b_{l^{\prime}} \geq 4 b_{l}$, whence $b_{l^{\prime}}>b_{l}+b_{k}+u$ because $b_{k} \leq b_{l}$ and $u \leq \Delta_{l}^{0} \leq b_{l}$. Thus, the equation $b_{l}+b_{k}+u=b_{l^{\prime}}+b_{k^{\prime}}+v$ cannot be satisfied.

Since $P_{n}=\mathbf{1}$ for all $n \in \mathbb{N} \backslash I$, it follows from Fact 1 that the compatibility equation can now be rewritten as

$$
\phi\left(T^{b_{k}+u} e_{0}\right)=\phi\left(T^{b_{k^{\prime}}+v} e_{0}\right) .
$$

Fact 2. Let $r \in \mathbb{N}$ be defined by $p \in[\beta(r), \beta(r+1))$. Then $\Delta_{l}^{1}=r=\Delta_{l^{\prime}}^{1}$, and $\Delta_{l}^{0}=\Delta_{l^{\prime}}^{0} \leq r+1$.

This is obvious by the definition of $\Delta_{l}^{i}$ and since $\alpha(r+2) \geq \beta(r+1)$.

Fact 3. We have $b_{k}-b_{k^{\prime}}>b_{q_{\theta(r)}}$. In particular, $k>k^{\prime}$ and $k>q_{\theta(r)}$.

Proof of Fact 3. By Fact 2, we have $b_{k}-b_{k^{\prime}} \geq b_{l^{\prime}}-b_{l}-u \geq b_{l^{\prime}}-b_{l}-r-1$. Moreover, since $q_{p}<l<l^{\prime}$, it follows from (4) in Lemma 4.1 that $b_{l^{\prime}}-b_{l}$ is a nonzero multiple of $2^{p+1}$, whence $b_{l^{\prime}}-b_{l} \geq 2^{\beta(r)+1}>r+1+b_{q_{\theta(r)}}$.

We now distinguish two cases.

Case 1. $k^{\prime}>q_{\alpha(r)}$.

In that case, $k$ and $k^{\prime}$ are both nonzero, so that $u, v \leq r$ by Fact 2 . From the equation $b_{l}+b_{k}+u=b_{l^{\prime}}+b_{k^{\prime}}+v$, Fact 3 and property (4) in Lemma 4.1, we get

$$
u \equiv v \bmod 2^{\alpha(r)+1} \text {. }
$$

Since $|u-v| \leq r$ and $2^{\alpha(r)+1}>r$, it follows that $u=v$, hence $b_{l}+b_{k}=b_{l^{\prime}}+b_{k^{\prime}}$. Now $l, l^{\prime}$ does not belong to $I$ and Lemma 4.1 ensures that $b_{k} \equiv b_{k^{\prime}}(\bmod 3)$. By Lemma 4.1 again and since $k, k^{\prime} \neq 0$, this implies that either $k$ and $k^{\prime}$ both belong to $I$, or they both belong to $\mathbb{N} \backslash I$. If $k, k^{\prime} \in I$, then $k=q_{m}$ and $k^{\prime}=q_{m^{\prime}}$, where $0<m^{\prime}<m \leq p$. Applying 4.1 once more, we get $b_{l}, b_{l^{\prime}}, b_{k} \equiv 0\left(\bmod 2^{m^{\prime}+1}\right)$ and 
$b_{k^{\prime}} \equiv 2^{m^{\prime}}\left(\bmod 2^{m^{\prime}+1}\right)$, which is impossible since $b_{l}+b_{k}=b_{l^{\prime}}+b_{k^{\prime}}$. Thus, $k$ and $k^{\prime}$ both belong to $\mathbb{N} \backslash I$, whence $P_{k}=\mathbf{1}=P_{k^{\prime}}$. Moreover, since $k>k^{\prime}>q_{\alpha(r)}$, we have $\Delta_{k}^{0}, \Delta_{k^{\prime}}^{0} \geq r$, and since $u, v \leq r$, it follows that $b_{k}+u \in \mathbf{J}_{k}$ and $b_{k^{\prime}}+v \in \mathbf{J}_{k^{\prime}}$. Therefore, we get by the induction hypothesis

$$
\phi\left(T^{b_{k}+u} e_{0}\right)=\phi\left(T^{u} e_{0}\right)=\phi\left(T^{v} e_{0}\right)=\phi\left(T^{b_{k^{\prime}}+v} e_{0}\right) .
$$

Case 2. $k^{\prime} \leq q_{\alpha(r)}$.

Since $l, l^{\prime}>q_{\beta(r)}$, we have $b_{l}, b_{l}^{\prime} \equiv 0\left(\bmod 2^{\beta(r)+1}\right)$; and since $k>q_{\theta(r)}$ by Fact 3 , we have $b_{k} \equiv 0\left(\bmod 2^{\theta(r)+1}\right)$. From the equation $b_{l}+b_{k}+u=b_{l^{\prime}}+b_{k^{\prime}}+v$ and since $\beta(r), \theta(r) \geq b_{q_{\alpha(r)}}$, it follows that

$$
u \equiv b_{k^{\prime}}+v\left(\bmod 2^{b_{q_{\alpha(r)}+1}}\right) .
$$

But $b_{k^{\prime}} \leq b_{q_{\alpha(r)}}$ and $u, v \leq r+1 \leq b_{q_{\alpha(r)}}+1$, so that $\left|b_{k^{\prime}}+v-u\right| \leq 2 b_{q_{\alpha(r)}}+1<$ $2^{b_{\alpha(r)}+1}$. Therefore, we have in fact $u=b_{k^{\prime}}+v$.

Looking at the equation $b_{l}+b_{k}=b_{l^{\prime}}$ modulo 3 , we see that $k \in \mathbb{N} \backslash I$, whence $P_{k}=1$. Moreover, since $k>q_{\theta(r)} \geq q_{\alpha(r)}$, we have $\Delta_{k}^{0} \geq r$. Finally, since $k$ is nonzero, we have $u \leq \Delta_{l}^{1}$, i.e. $u \leq r$ by Fact 2 . Hence $u \leq \Delta_{k}^{0}$, so that $u \in \mathbf{J}_{k}$. Therefore, we get

$$
\phi\left(T^{b_{k}+u} e_{0}\right)=\phi\left(T^{u} e_{0}\right)=\phi\left(T^{b_{k^{\prime}}+v} e_{0}\right) .
$$

This concludes the proof of Lemma 4.2

4.3. Proof of Theorem 1.2. The sequence $\left(b_{n}\right)$ clearly satisfies $\lim _{n \rightarrow \infty}\left(b_{n}-b_{n-1}\right)=$ $\infty$, and we have just proved that one can find some pair $\Delta$ of nondecreasing sequences tending to $\infty$ such that the $\boldsymbol{\Delta}$-compatible linear functional $\phi$ is well-defined. Since we are working on $\ell^{1}(\mathbb{N})$, we can then use Lemmas 3.1 and 2.4 exactly as in the proof of Theorem 1.6 to conclude that for a suitable choice of parameters, the operator $T$ extends to a continuous, non-weakly-mixing operator on $X$ for which the set $\mathbf{B}=\left\{b_{n} ; n \in \mathbb{N} \backslash I\right\}$ is a hypercyclicity set.

The only point that remains to be checked is that the increasing enumeration of any cofinite subset of $\mathbf{B}$ does not grow faster than $\left(m_{k}\right)$. But such an enumeration has the form $\left(b_{j_{k}}\right)$, where $j_{k} \leq 2 k$ for large $k$ if the sequence $\left(q_{p}\right)$ is sufficiently fast increasing. Thus, the result is clear since we know that $b_{2 k}=O\left(m_{k}\right)$.

\section{MORE ON HYPERCYCLICITY SETS}

In this section, we collect some further results on hypercyclicity sets.

5.1. A criterion for B-hypercyclicity. In the spirit of [BaGr, Thm 2.1] and [BonGE, Thm 2.1], one can give a criterion for an operator to admit a given set $\mathbf{B} \subset \mathbb{N}$ as a hypercyclicity set. Our setting is a separable $F$-space $X$ endowed with a translation-invariant metric $d$. For notational simplicity, we set $\|x\|:=d(x, 0)$. 
Theorem 5.1. Let $\left(b_{n}\right)$ be an increasing sequence of integers such that $b_{n+1}-b_{n} \rightarrow$ $\infty$, and let $T \in \mathfrak{L}(X)$. Assume that there exist a dense set $D \subset X$ and a map $S: D \rightarrow D$ such that:

(1) $\lim _{i \rightarrow \infty} \sup _{J \subset[0, i)}\left\|\sum_{k \in J} T^{b_{i}-b_{k}}(x)\right\|=0$ for all $x \in D$;

(2) for any $x \in D$ and every $\varepsilon>0$, one can find $A=A(x, \varepsilon)>0$ such that whenever $i \leq j<j^{\prime}$ and $b_{j}-b_{i} \geq A$, one has

$$
\left\|\sum_{j \leq k \leq j^{\prime}} S^{b_{k}-b_{i}}(x)\right\|<\varepsilon
$$

(3) $T S(x)=x$ for all $x \in D$.

Then $\mathbf{B}=\left\{b_{n} ; n \in \mathbb{N}\right\}$ is a hypercyclicity set for $T$.

Proof. The operator $T$ is hypercyclic, because it satisfies the hypercyclicity criterion with respect to the sequence $\left(b_{k+1}-b_{k}\right)$. Let us fix a vector $z \in H C(T)$. To show that $\mathbf{B}$ is a hypercyclicity set for $T$, it is enough to find some vector $x \in X$ such that $T^{b_{i}}(x) \rightarrow z$.

First, we note that, replacing $b_{n}$ by $b_{n}-b_{0}$ and $z$ by $T^{b_{0}}(z)$, which is also a hypercyclic vector for $T$, we may and do assume that $b_{0}=0$.

Let $\left(x_{p}\right)_{p \in \mathbb{N}}$ be a sequence in $D$ such that $\left\|x_{p}-z\right\|<2^{-p}$ for all $p$. Then, let us choose an increasing sequence of integers $\left(i_{p}\right)$ such that

$$
\begin{gathered}
k \geq i_{p} \Longrightarrow b_{k}-b_{k-1} \geq A\left(x_{p}, 2^{-p}\right) ; \\
\forall i \geq i_{p} \sum_{q=1}^{p} \sup _{J \subset[0, i)}\left\|\sum_{k \in J} T^{b_{i}-b_{k}}\left(x_{q}\right)\right\|<2^{-p} .
\end{gathered}
$$

Finally, let us define a sequence $\left(y_{k}\right)_{k \in \mathbb{N}}$ by putting $y_{k}:=x_{p}$ for $k \in\left[i_{p}, i_{p+1}\right)$, and set $x:=\sum_{k=0}^{\infty} S^{b_{k}}\left(y_{k}\right)$. This series is indeed convergent, because if $i_{p} \leq j<j^{\prime}$, then we get by $(2)$

$$
\left\|\sum_{j \leq k \leq j^{\prime}} S^{b_{k}}\left(y_{k}\right)\right\| \leq \sum_{q=p}^{\infty}\left\|\sum_{\left[i_{q}, i_{q+1}\right) \cap\left[j, j^{\prime}\right]} S^{b_{k}-b_{0}}\left(x_{q}\right)\right\| \leq 2^{-p+1} .
$$

If $i \in \mathbb{N}$ and $i_{p} \leq i<i_{p+1}$, then

$$
\left\|T^{b_{i}}(x)-z\right\| \leq\left\|\sum_{k<i} T^{b_{i}-b_{k}}\left(y_{k}\right)\right\|+2^{-p}+\left\|\sum_{k>i} S^{b_{k}-b_{i}}\left(y_{k}\right)\right\| .
$$

The first term in the right-hand side is easily controlled by

$$
\sum_{q=1}^{p}\left\|\sum_{\left[i_{q}, i_{q+1} \wedge i\right)} T^{b_{i}-b_{k}}\left(x_{q}\right)\right\|<2^{-p} .
$$


For the third term, we write

$$
\begin{aligned}
\left\|\sum_{k>i} S^{b_{k}-b_{i}}\left(y_{k}\right)\right\| & \leq \sum_{q=p}^{\infty}\left\|\sum_{(i, \infty) \cap\left[i_{q}, i_{q+1}\right)} S^{b_{k}-b_{i}}\left(x_{q}\right)\right\| \\
& \leq 2^{-p} .
\end{aligned}
$$

Thus, we have shown that $T^{b_{i}}(x) \rightarrow z$ as $i \rightarrow \infty$, which concludes the proof.

Corollary 5.2. Let $T \in \mathfrak{L}(X)$. Assume there exist $D \subset X$ dense and $S: D \rightarrow D$ satisfying, for any $x \in D$ :

(1) $\sum_{n \geq 0}\left\|T^{n} x\right\|<+\infty$;

(2) $\sum_{n \geq 0}\left\|S^{n} x\right\|<+\infty$;

(3) $T S(x)=x$.

Then any sequence $\left(b_{n}\right)$ such that $b_{n+1}-b_{n} \rightarrow \infty$ defines a hypercyclicity set for $T$.

Observe that an operator which satisfies the previous assumptions is in fact frequently hypercyclic (see [BaGr, Theorem 2.1]). Notice also that even in that case, one must assume that $b_{n+1}-b_{n} \rightarrow \infty$; see Theorem 5.4 below.

Example 5.3. Let $B$ be the Bergman backward shift. For any $\delta>0$, the set $\mathbf{B}_{\delta}:=\left\{\left[n^{1+\delta}\right] ; n \in \mathbb{N}\right\}$ is a hypercyclicity set for $B$. Yet, $B$ is not frequently hypercyclic.

Proof. The operator $B$ can be defined as the backward shift on $\ell^{2}(\mathbb{N})$ with weights $\omega_{i}=\sqrt{\frac{i+1}{i}}$. That is, $B\left(e_{0}\right)=0$ and $B\left(e_{i}\right)=\omega_{i} e_{i-1}$ for any $i \geq 1$, where $\left(e_{i}\right)$ is the canonical basis of $\ell^{2}(\mathbb{N})$. It is proved in [BaGr, Example 2.9] that $B$ is not frequently hypercyclic. We fix $\delta>0$, and we apply 5.1 to show that $\mathbf{B}_{\delta}$ is a hypercyclicity set for $B$.

The operator $S$ will be the forward shift defined by $S\left(e_{i}\right)=\frac{1}{\omega_{i+1}} e_{i+1}$ and we take as $D$ the set of finitely supported sequences $c_{00}$. Since $D=\bigcup_{n \in \mathbb{N}} \operatorname{Ker}\left(B^{n}\right)$, condition (1) in Theorem 5.1 is clearly satisfied, so we just have to prove that (2) holds for any $x \in D$. We do that for $x=e_{0}$, the proof for an arbitrary $x \in D$ being the same.

Since $\omega_{1} \cdots \omega_{n}=\sqrt{n+1}$, we have $S^{n}\left(e_{0}\right)=\sqrt{\frac{1}{n+1}} e_{n}$ for all $n \geq 1$. Thus, we must show that for any $\varepsilon>0$, there exists $A>0$ such that, whenever $i, j \in \mathbb{N}$ and $\left[j^{1+\delta}\right]-\left[i^{1+\delta}\right] \geq A$, one has

$$
\sum_{k \geq j} \frac{1}{\left[k^{1+\delta}\right]-\left[i^{1+\delta}\right]}<\varepsilon .
$$

This follows from the elementary result

$$
\lim _{j \rightarrow+\infty} \sum_{k>j} \frac{1}{k^{1+\delta}-j^{1+\delta}}=0 .
$$


To obtain that, we write

$$
\begin{aligned}
\sum_{k>j} \frac{1}{k^{1+\delta}-j^{1+\delta}} & =\sum_{k>j} \sum_{m \geq 0} \frac{j^{(1+\delta) m}}{k^{(1+\delta)(m+1)}} \\
& \leq \sum_{m \geq 0} j^{(1+\delta) m}\left(\frac{1}{(j+1)^{(1+\delta)(m+1)}}+\int_{j+1}^{\infty} \frac{d t}{t^{(1+\delta)(m+1)}}\right) \\
& \leq \frac{1}{(j+1)^{1+\delta}-j^{1+\delta}}+\frac{1}{\delta j^{\delta}} \sum_{m \geq 0} \frac{1}{m+1}\left(\frac{j}{j+1}\right)^{(1+\delta) m} \\
& \leq \frac{1}{(j+1)^{1+\delta}-j^{1+\delta}}+2 \frac{\log (j+1)}{\delta j^{\delta}}
\end{aligned}
$$

5.2. Which sets are hypercyclicity sets. Using Theorem 5.1, it is now very easy to determine which sets of natural numbers can be hypercyclicity sets for some (perhaps weakly mixing) operator.

Theorem 5.4. Let $\mathbf{B}$ be an infinite subset of $\mathbb{N}$, and let $\left(b_{n}\right)$ be the increasing enumeration of $\mathbf{B}$. The following are equivalent.

(1) $\mathbf{B}$ is a hypercyclicity set for some $F$-space operator.

(2) $\mathbf{B}$ is a hypercyclicity set for $T=2 B$, where $B$ is the usual backward shift on $\ell^{2}(\mathbb{N})$.

(3) $\lim _{n \rightarrow \infty}\left(b_{n+1}-b_{n}\right)=\infty$.

Proof. That (3) implies (2) follows Corollary 5.2, so it remains to show that (1) implies (3). Assume that $\mathbf{B}$ is a hypercyclicity set for some operator $T \in \mathcal{L}(X)$, and that $b_{j+1}-b_{j} \leq M$ for some finite constant $M$ and infinitely many $j$. Choose $x \in X$ such that each set $\mathbf{N}(x, V)$ contains a translate of some cofinite subset of $\mathbf{B}$. Then $x$ cannot be a periodic point for $T$, so one can find a neighbourhood $U$ of $x$ such that $T^{r}(U) \cap U=\varnothing$ for $r=0, \ldots, M$. By the choice of $x$, there exist $a, j_{0} \in \mathbb{N}$ such that $T^{b_{j}+a}(x) \in U$ for all $j \geq j_{0}$. Writing $b_{j+1}-b_{j}=\left(a+b_{j+1}\right)-\left(a+b_{j}\right)$, it follows that $T^{b_{j+1}-b_{j}}(U) \cap U \neq \varnothing$ for any $j \geq j_{0}$. Since $b_{j+1}-b_{j} \leq M$ for infinitely many $j$, this contradicts the choice of $U$.

5.3. Weak mixing and difference sets. By Theorem 5.4, being a hypercyclicity set for some operator $T$ is just a matter of growth. On the other hand, as we already observed in the very beginning of the paper, the situation is quite different if one requires $T$ to be non-weakly mixing. We restate here the result from [GEP] mentionned in the introduction. Recall that an infinite set of natural numbers $\mathbf{N}$ is said to be syndetic (or to have bounded gaps) if there exists some fixed $K>0$ such that $\mathbf{N}$ meets each interval of length $K$; equivalently, if $\mathbf{N}$ can be enumerated as an increasing sequence $\left(n_{k}\right)$ with $\sup _{k}\left(n_{k+1}-n_{k}\right)<\infty$. 
Proposition 5.5. Let $T \in \mathfrak{L}(X)$. Assume one can find $x \in X$ such that for every nonempty open set $V \subset X$, the difference set $\mathbf{N}(x, V)-\mathbf{N}(x, V)$ is syndetic. Then $T$ is weakly mixing.

As an immediate consequence, we get:

Corollary 5.6. Let $\mathbf{B}$ be an infinite subset of $\mathbb{N}$ whose difference set $\mathbf{B}-\mathbf{B}$ is syndetic. Then $\mathbf{B}$ cannot be a hypercyclicity set for any non-weakly mixing operator.

This clearly illustrate the well-established fact weak mixing is closely related to arithmetical properties of sets of integers. It also points out that the operator constructed in Section 2 can be weakly mixing. Indeed, if we take $X=\ell^{1}(\mathbb{N}), b_{n}=n^{2}$ and $I=2 \mathbb{N}$, then we can arrange $\left(P_{n}\right)$ and $\left(a_{n}\right)$ so that $T$ is a continuous hypercyclic operator with $\left((2 n+1)^{2}\right)$ as a hypercyclicity sequence. From Corollary 5.6, we deduce that $T$ is weakly mixing.

\section{SOME NON-BANACH EXAMPLES}

It is proved in $[\mathrm{BaM}]$ that hypercyclic non-weakly mixing operators can be constructed on the space of entire functions $\mathcal{H}(\mathbb{C})$. We were not able to show that Theorem 1.2 holds in $\mathcal{H}(\mathbb{C})$. However, we will now show that our methods still give some results in a Fréchet space setting.

We will consider sequences spaces of the form

$$
X_{\mathrm{w}}:=\left\{x=\left(x_{i}\right) \in \mathbb{K}^{\mathbb{N}} ; \forall r \geq 0 \quad N_{r}(x):=\sum_{i=0}^{\infty} w(i)^{r}\left|x_{i}\right|<\infty\right\},
$$

where $\mathbf{w}=(w(i))_{i \in \mathbb{N}}$ is an increasing sequence of positive numbers tending to $\infty$. The space $X_{\mathrm{w}}$ is endowed with the topology generated by the norms $N_{r}, r \geq 0$.

For example, the space of entire functions $\mathcal{H}(\mathbb{C})$ can be identified with the space $X_{\mathbf{w}}$ where $w(i)=e^{i}$; and if we take $w(i)=i+1$, then we get the space

$$
A^{\infty}(\mathbb{T}):=\mathcal{H}(\mathbb{D}) \cap \mathcal{C}^{\infty}(\mathbb{T}) .
$$

As usual, we denote by $\left(e_{i}\right)_{i \in \mathbb{N}}$ the canonical basis of $X_{\mathbf{w}}$, and by $c_{00}$ the linear span of the $e_{i}$ 's. The next lemma shows that, as far as the continuity of linear or bilinear maps is concerned, the space $X_{\mathrm{w}}$ behaves more or less like $\ell^{1}(\mathbb{N})$.

Lemma 6.1. Let $c_{00}$ be equipped with the topology induced by $X_{\mathbf{w}}$.

(a) Let $R: c_{00} \rightarrow c_{00}$ be a linear map such that $R\left(e_{i}\right) \in \operatorname{span}\left\{e_{j} ; j \leq i\right\}$ for all $i \in \mathbb{N}$. If the sequence $\left(R\left(e_{i}\right)\right)$ is $\|\cdot\|_{1}$-bounded, then $R$ is continuous.

(b) If $B: c_{00} \times c_{00} \rightarrow \mathbb{K}$ is a bilinear functional such that the double sequence $\left(B\left(e_{p}, e_{q}\right)\right)$ is bounded, then $B$ is continuous. 
Proof. Part (b) is obvious because $B$ is continuous with respect to the the $\ell^{1}$-norm and $\|\cdot\|_{1}=N_{0}$. To prove (a), observe that since the sequence $\mathbf{w}$ is increasing, we have $N_{r}(z) \leq w(i)^{r}\|z\|_{1}$ for each $i \in \mathbb{N}$ and all $z \in \operatorname{span}\left\{e_{j} ; j \leq i\right\}$. Setting $C:=\sup _{i}\left\|R\left(e_{i}\right)\right\|_{1}$, it follows that $N_{r}(R(x)) \leq C N_{r}(x)$ for each $r$ and all $x \in c_{00}$.

¿From now on, we will only consider sequences $\mathbf{w}$ such that $\sup _{i \in \mathbb{N}} \frac{w(i+1)}{w(i)}<\infty$. This property ensures that the forward shift $S$ with weight sequence $(w(i))_{i \geq 1}$ is continuous on $X_{\mathrm{w}}$. Recall that $S$ is the linear map defined on $c_{00}$ by the identities $S\left(e_{i}\right)=w(i+1) e_{i+1}$.

In the present setting, the "linear map with parameters" $T: c_{00} \rightarrow c_{00}$ is still welldefined, but there are two important changes. The first one is that the space on which $T$ is supposed to act becomes itself a parameter, since it is defined by the sequence $\mathbf{w}$; and of course, $w(i)$ has no longer the form $K(1-\xi(i))$. The second change is that, since we require $\frac{1}{a_{n}} e_{b_{n}}$ to tend to 0 for the topology of $X_{\mathbf{w}}$, the sequence $\left(a_{n}\right)$ can no longer be chosen independently of $b_{n}$. We will assume that $\left(a_{n}\right)$ has the form

$$
a_{n}=c w\left(b_{n}\right)^{r_{n}},
$$

for some constant $c>0$ and some nondecreasing sequence of natural numbers $\left(r_{n}\right)$ tending to $\infty$.

In view of Lemma 6.1, all we have to do if we want to show that $T$ extends to a non-weakly mixing operator on $X_{\mathbf{w}}$ having $\mathbf{B}:=\left\{b_{n} ; n \in \mathbb{N} \backslash I\right\}$ as a hypercyclicity set, is to check that (i) the sequence $\left(f_{n}\right)$ is $\|\cdot\|_{1}$-bounded; and (ii) one can construct as usual a linear functional $\phi$ such that the double sequence $\left(\left(\phi\left(e_{p} \cdot e_{q}\right)\right)\right.$ is bounded.

Proposition 6.2. Let $\left(m_{k}\right)$ be an increasing sequence of natural numbers such that $m_{k} / k \rightarrow \infty$. If the sequence $\mathbf{w}$ is sufficiently slowly increasing, then there exists a non-weakly mixing, $\left(m_{k}\right)$-hypercyclic operator on $X_{\mathbf{w}}$.

Proof. Let $\left(b_{n}\right)$ and $I=\left\{q_{p} ; p \in \mathbb{N}\right\}$ be the increasing sequence of integers and the infinite set given by Lemma 4.1. Let also $\boldsymbol{\Delta}=\left(\left(\Delta_{l}^{0}\right),\left(\Delta_{l}^{1}\right)\right)$ be the pair of nondecreasing sequences of natural numbers given by Lemma 4.2. We show that if the sequences $\left(\left|P_{n}\right|_{1}\right),\left(d_{n}\right)$ and $\left(r_{n}\right)$ are suitably controlled, then our operator $T$ and the map $(x, y) \mapsto \phi(x \cdot y)$ are continuous.

The proof of Lemma 2.2 has shown that if $n \in \mathbb{N}$ and $\left\|f_{k}\right\|_{1} \leq 1$ for all $k<n$, then (with the usual notation $D_{n}=b_{n}-b_{n-1}$ )

$$
\left\|f_{n}\right\|_{1} \leq\left|a_{n-1}\right|\left|P_{n}\right|_{1} \frac{\prod_{i=1}^{d_{n}} w(i)}{\prod_{i=1}^{D_{n}-1} w\left(i+b_{n-1}\right)}+\left|a_{n}\right|\left|P_{n-1}\right|_{1} \frac{\prod_{i=1}^{D_{n}+d_{n-1}} w(i)}{\prod_{i=1}^{D_{n}-1} w\left(i+b_{n-1}\right)} .
$$

Assume that $b_{n-1} \geq d_{n}$. Then, since $a_{n-1}=c w\left(b_{n-1}\right)^{r_{n-1}}$ and since $\mathbf{w}$ is increasing, the first term $\mathbf{I}$ in the right-hand side of the above inequality is not greater than

$$
c\left|P_{n}\right|_{1} w\left(b_{n-1}\right)^{-\left(D_{n}-1-r_{n-1}-d_{n}\right)} .
$$


To estimate the second term II, we write it as

$$
c\left|P_{n-1}\right|_{1} \frac{w\left(b_{n}\right)^{r_{n}}}{\prod_{i=b_{n}-r_{n}}^{b_{n}-1} w(i)} \frac{\prod_{i=1}^{D_{n}+d_{n-1}} w(i)}{\prod_{i=1}^{D_{n}-2 r_{n}-1} w\left(i+b_{n-1}\right)} \frac{1}{\prod_{i=b_{n}-2 r_{n}}^{b_{n}-r_{n}-1} w(i)} .
$$

We know that $\mathbf{w}$ is increasing with $w(i+1) \leq C w(i)$ for some fixed constant $C<\infty$. Thus, we have $w\left(b_{n}-j\right) \geq C^{-j} w\left(b_{n}\right)$ for all $j \leq b_{n}$. If we assume that $b_{n-1} \geq$ $d_{n-1}+2 r_{n}+1$, it follows that

$$
\begin{aligned}
\text { II } & \leq c\left|P_{n-1}\right|_{1} \times C^{1+\cdots+r_{n}} \times \prod_{i=1}^{\left(d_{n-1}+2 r_{n}+1\right)} w(i) \times \frac{C^{\left(r_{n}+1\right)+\cdots+2 r_{n}}}{w\left(b_{n}\right)^{r_{n}}} \\
& \leq c\left|P_{n-1}\right|_{1} \frac{w\left(d_{n-1}+2 r_{n}+1\right)^{d_{n-1}+2 r_{n}+1} C^{4 r_{n}^{2}}}{w\left(b_{n}\right)^{r_{n}}} .
\end{aligned}
$$

Since $D_{n} \rightarrow \infty$ and $w\left(b_{n}\right) \rightarrow \infty$, it is now clear that if $c$ is small enough to take care of the first $f_{n}$ 's and if the sequences $\left(P_{n}\right)$ and $\left(r_{n}\right)$ are suitably controlled by some sequence $\left(u_{n}\right)$ tending to $\infty$, then the sequence $\left(f_{n}\right)$ is $\|\cdot\|_{1}$-bounded.

Now, let $\phi$ be the $\Delta$-compatible linear functional, and set $\Delta_{l}=\min \left(\Delta_{l}^{0}, \Delta_{l}^{1}\right)$. If $p \leq q \in\left[b_{l}, b_{l+1}\right)$, then, by the remark following Lemma 2.4 and since $a_{l}=w\left(b_{l}\right)^{r_{l}}$, we have

$$
\left|\phi\left(e_{p} \cdot e_{q}\right)\right| \leq 4 \prod_{j=1}^{2 b_{l}+1} \max \left(1,\left|P_{j}\right|_{1}\right) \times \frac{w\left(b_{l}\right)^{2 r_{l}}}{w\left(b_{i(l)}\right)^{\Delta_{l} / 4}},
$$

where $i(l):=\min \left\{i ; b_{i+1}-b_{i}>\frac{\Delta_{l}}{4}\right\}$. Since $i(l) \rightarrow \infty$, it follows that if $\mathbf{w}$ is sufficiently slowly increasing, then one can choose $\left(r_{l}\right)$ in such a way that the double sequence $\left(\phi\left(e_{p} \cdot e_{q}\right)\right)$ is bounded.

Proposition 6.3. Assume that the sequence $\mathbf{w}$ has at most a polynomial growth and satisfies $\liminf \frac{w(i)}{i^{\beta}}>0$ for some $\beta>0$. Then for any $\varepsilon>0$, there exists a non-weakly mixing, $\left(k^{3+\varepsilon}\right)$-hypercyclic operator on $X_{\mathbf{w}}$.

Proof. By Lemma 3.2, one can find an increasing sequence $\left(b_{n}\right)$ which is $\left(\left[l^{\alpha}\right]\right)$-Sidon for some $\alpha>0$ and satisfies $b_{n}=O\left(n^{3+\varepsilon}\right)$. Moreover, one can also require that $b_{n} \geq n^{3+\varepsilon}$ for all $n$ (see Remark 2 just after 3.2 ); in particular, we have $b_{n+1}=O\left(b_{n}\right)$. The proof of Proposition 6.2 has established that the operator $T$ associated to $\left(b_{n}\right)$ and $I:=2 \mathbb{N}$ is continuous if the sequences $\left(r_{n}\right)$ and $\left(P_{n}\right)$ are suitably controlled. Therefore (looking at the proof of 6.2 again), the only thing that remains to be done is to show that if the sequence $\left(r_{n}\right)$ is suitably chosen, then

$$
\tau_{l}:=\frac{w\left(b_{l}\right)^{2 r_{l}}}{w\left(b_{i(l)}\right)^{\Delta_{l} / 4}} \rightarrow 0 \quad \text { as } l \rightarrow \infty
$$


where $\Delta_{l}=\left[l^{\alpha}\right]$. Now, we have $b_{i(l)+1} \geq b_{i(l)+1}-b_{i(l)}>\frac{\left[l^{\alpha}\right]}{4}$ by definition of $i(l)$, and since $b_{i(l)+1}=O\left(b_{i(l)}\right)$ we get $\liminf \frac{b_{i(l)}}{\left[l^{\alpha}\right]}>0$. Since $\liminf \frac{w(i)}{i^{\beta}}>0$ and both $\mathbf{w}$ and $\left(b_{l}\right)$ have polynomial growth, it follows that

$$
\tau_{l} \leq \frac{l^{C r_{l}}}{\left[l^{\alpha}\right]^{c\left[l^{\alpha}\right]}}=\exp \left(C r_{l} \log l-c\left[l^{\alpha}\right] \log \left[l^{\alpha}\right]\right),
$$

for some constants $c>0$ and $C<\infty$. Thus, one can clearly choose $\left(r_{l}\right)$ so that $\tau_{l} \rightarrow 0$.

Corollary 6.4. There exists a non-weakly mixing, $\left(k^{3+\varepsilon}\right)$-hypercyclic operator on $A^{\infty}(\mathbb{T})$.

Proof. The space $A^{\infty}(\mathbb{T})$ can be identified with $X_{\mathbf{w}}$, where $w(i)=i+1$.

Remark. Unfortunately, the above proof breaks down in the space of entire functions $\mathcal{H}(\mathbb{C})$, which corresponds to $w(i)=e^{i}$.

\section{SETS OF INTEGERS}

It has been known for quite a long time that there are strong connections between topological dynamics and some natural classes of subsets of $\mathbb{N}$; see e.g. [F] or [GlW]. The aim of this final section is to briefly illustrate the usefulness of this point of view in the linear setting as well.

For the sake of clarity, we will not assume from the beginning that we are dealing with linear maps. The most general setting is that of a continuous map $T: X \rightarrow X$ acting on some Hausdorff topological space $X$. Then hypercyclicity, topological transitivity and weak mixing make sense with the same definitions. Topological transitivity implies hypercyclicity if $X$ is a second countable Baire space, and the converse implication holds if $X$ has no isolated point. We will assume that $X$ is separable, completely metrizable and without isolated points.

7.1. Some notations. For any point $x \in X$ and any nonempty open set $V \subset X$, we set as usual

$$
\mathbf{N}(x, V)=\left\{n \in \mathbb{N} ; T^{n}(x) \in V\right\} .
$$

If $V$ and $V^{\prime}$ are nonempty open subsets of $X$, we set

$$
\mathbf{N}\left(V, V^{\prime}\right)=\left\{n ; T^{n}(V) \cap V^{\prime} \neq \varnothing\right\},
$$

and we also define

$$
\mathbf{C}\left(V, V^{\prime}\right)=\left\{n ; T^{n}(V) \subset V^{\prime}\right\} .
$$

If $\mathbf{N}$ and $\mathbf{N}^{\prime}$ are two subsets of $\mathbb{N}$, the difference-set $\mathbf{N}^{\prime}-\mathbf{N}$ is defined by

$$
\mathbf{N}^{\prime}-\mathbf{N}=\left\{n^{\prime}-n ;\left(n, n^{\prime}\right) \in \mathbf{N} \times \mathbf{N}^{\prime}, n^{\prime} \geq n\right\},
$$

and the sum-set $\mathbf{N}+\mathbf{N}^{\prime}$ is defined in the obvious way. 
It is easy to check that if $x \in X$ is a hypercyclic point for $T$, then

$$
\mathbf{N}\left(V, V^{\prime}\right)=\mathbf{N}\left(x, V^{\prime}\right)-\mathbf{N}(x, V)
$$

for any open sets $V, V^{\prime} \subset X$.

Finally, the following identies are also easily verified, for any open sets $A, B, C$ :

$$
\begin{aligned}
& \mathbf{N}(A, B)+\mathbf{C}(B, C) \subset \mathbf{N}(A, C), \\
& \mathbf{N}(A, C)-\mathbf{C}(A, B) \subset \mathbf{N}(B, C) .
\end{aligned}
$$

7.2. Back to the linear functional $\phi$. The statement of Lemma 2.1 may look a bit strange, since the continuity property of the linear functional $\phi$ does not seem very intuitive. We intend to show here that properties of that kind are in fact very natural when dealing with weak mixing. In what follows, we denote by $O_{T}(x)$ the $T$-orbit of a point $x \in X$; that is $O_{T}(x)=\left\{T^{n}(x) ; n \in \mathbb{N}\right\}$.

Observe that if $\phi$ is a linear functional as 2.1, then one can find two nonempty open sets $\mathcal{W}, \mathcal{W}^{\prime}$ in $O_{T}\left(e_{0}\right) \times O_{T}\left(e_{0}\right)$ such that the sets $\{x \cdot y ;(x, y) \in \mathcal{W}\}$ and $\left\{x^{\prime} \cdot y^{\prime} ;\left(x^{\prime}, y^{\prime}\right) \in \mathcal{W}^{\prime}\right\}$ are disjoint: just set $\mathcal{W}:=\{(u, v) ;|\phi(u \cdot v)|<1\}$ and $\mathcal{W}^{\prime}:=$ $\{(u, v) ;|\phi(u \cdot v)|>1\}$. Now, one can find four nonempty open sets $V_{1}, V_{2}, V_{3}, V_{4} \subset X$ such that $W \supset\left(V_{1} \times V_{4}\right) \cap\left(O_{T}\left(e_{0}\right) \times O_{T}\left(e_{0}\right)\right)$ and $W^{\prime} \supset\left(V_{2} \times V_{3}\right) \cap\left(O_{T}\left(e_{0}\right) \times O_{T}\left(e_{0}\right)\right)$, so that the above information may be translated purely in terms of sets of integers, as follows: $\left(\mathbf{N}\left(e_{0}, V_{1}\right)+\mathbf{N}\left(e_{0}, V_{4}\right)\right) \cap\left(\mathbf{N}\left(e_{0}, V_{3}\right)+\mathbf{N}\left(e_{0}, V_{2}\right)\right)=\varnothing$. This has in fact nothing to do with the linearity of $\phi$ : it is enough to have some nonconstant map $f: O_{T}\left(e_{0}\right) \rightarrow Y$, where $Y$ is a Hausdorff topological space, such that the map $(x, y) \mapsto f(x \cdot y)$ is continuous.

In view of these remarks, Lemma 2.1 is now an immediate consequence of the next proposition, which also explains in some sense why Sidon-type properties come up naturally in the questions we are looking at.

Proposition 7.1. Assume that $T$ is hypercyclic with hypercyclic point $x$. The following are equivalent.

(1) $T$ is not weakly mixing.

(2) There exist nonempty open sets $V_{1}, V_{2}, V_{3}, V_{4} \subset X$ such that the equation $n_{1}+n_{4}=n_{3}+n_{2}$ has no solution $\left(n_{1}, n_{2}, n_{3}, n_{4}\right)$ with $n_{i} \in \mathbf{N}\left(x, V_{i}\right)$ and $n_{1} \leq n_{3}$.

If $X$ is an $F$-space, $T \in \mathcal{L}(X)$ and $T$ is moreover invertible, these properties are equivalent to

(2') The same as (2) without the restriction $n_{1} \leq n_{3}$ : there exist $V_{1}, \ldots, V_{4}$ with $\left(\mathbf{N}\left(x, V_{1}\right)+\mathbf{N}\left(x, V_{4}\right)\right) \cap\left(\mathbf{N}\left(x, V_{3}\right)+\mathbf{N}\left(x, V_{2}\right)\right)=\varnothing$.

Proof. By the identity $\mathbf{N}\left(V, V^{\prime}\right)=\mathbf{N}\left(x, V^{\prime}\right)-\mathbf{N}(x, V)$, condition (2) is equivalent to the existence of four nonempty open sets $V_{i}$ such that $\mathbf{N}\left(V_{1}, V_{3}\right) \cap \mathbf{N}\left(V_{2}, V_{4}\right)=\varnothing$; that is, $(T \times T)^{k}\left(V_{1} \times V_{2}\right) \cap V_{3} \times V_{4}=\varnothing$ for all $k \in \mathbb{N}$. This means exactly that $T \times T$ is not topologically transitive. 
Now, assume that $X$ is an $F$-space and that $T \in \mathcal{L}(X)$ is invertible and not weakly mixing. We claim that one can find nonempty open sets $V_{1}, V_{2}, V_{3}, V_{4} \subset X$ such that $(T \times T)^{k}\left(V_{1} \times V_{2}\right) \cap V_{3} \times V_{4}=\varnothing$ for all $k \in \mathbb{Z}$. Indeed, otherwise a Baire category argument gives a vector $z \in X \times X$ whose two-sided $(T \times T)$-orbit $\left\{(T \times T)^{k}(z) ; k \in \mathbb{Z}\right\}$ is dense in $X \times X$. By the Bourdon-Feldman Theorem ([BouF]) and since an invertible operator is hypercyclic if and only if its inverse is, it follows that $T \times T$ is hypercyclic, a contradiction. Replacing the sets $\mathbf{N}(x, V)$ and $\mathbf{N}\left(V, V^{\prime}\right)$ by the analogous subsets of $\mathbb{Z}$, we now get in the same way as above that $\left(2^{\prime}\right)$ is satisfied.

7.3. Thick sets and weak mixing. A set $\mathbf{B} \subset \mathbb{N}$ is said to be thick if it contains arbitrarily long intervals. Equivalently, $\mathbf{B}$ is thick iff it intersects every syndetic set.

Thick sets are always present (at least implicitely) when one is dealing with weak mixing. This is apparent in the following theorem, which collects several well-known characterizations of weak mixing.

Theorem 7.2. The following are equivalent.

(1) $T$ is weakly mixing.

(2) The sets $\mathbf{N}\left(V, V^{\prime}\right)$ form a filter basis: each $\mathbf{N}\left(V, V^{\prime}\right)$ is nonempty, and given $V_{1}, V_{1}^{\prime}, V_{2}, V_{2}^{\prime}$, one can find $V_{3}, V_{3}^{\prime}$ such that $\mathbf{N}\left(V_{3}, V_{3}^{\prime}\right) \subset \mathbf{N}\left(V_{1}, V_{1}^{\prime}\right) \cap \mathbf{N}\left(V_{2}, V_{2}^{\prime}\right)$

(3) For any $k \geq 1$, the $k$-fold product map $T \times \cdots \times T$ is topologically transitive.

(4) Each set $\mathbf{N}\left(V, V^{\prime}\right)$ is thick.

(5) For any $V, V^{\prime}$ and each $m \in \mathbb{N}$, one can find $k \in \mathbb{N}$ such that $T^{k}(V) \cap V^{\prime} \neq \varnothing$ and $T^{k+m}(V) \cap V^{\prime} \neq \varnothing$.

(6) For any $V, V_{1}, V_{2}$, one has $\mathbf{N}\left(V, V_{1}\right) \cap \mathbf{N}\left(V, V_{2}\right) \neq \varnothing$.

Proof. The implications $(1) \Rightarrow(2)$ and $(6) \Rightarrow(1)$ are proved in [Gl, Theorem 1.11], and the implications $(2) \Rightarrow \cdots \Rightarrow(5)$ are trivial. We show that (5) implies (6). Let $V, V_{1}, V_{2}$ be given. Since $T$ is topologically transitive, one can find $m \in \mathbb{N}$ and an open set $V^{\prime} \subset V_{1}$ such that $T^{m}\left(V^{\prime}\right) \subset V_{2}$. Applying (5), one gets $k \in \mathbb{N}$ such that $T^{k}(V) \cap V^{\prime} \neq \varnothing$ and $T^{k+m}(V) \cap V^{\prime} \neq \varnothing$. Then $k+m \in \mathbf{N}\left(V, V^{\prime}\right) \subset \mathbf{N}\left(V, V_{1}\right)$, and $k+m \in \mathbf{N}\left(V, V^{\prime}\right)+\mathbf{C}\left(V^{\prime}, V_{2}\right) \subset \mathbf{N}\left(V, V_{2}\right)$.

Natural examples of thick sets are provided by the following lemma.

Lemma 7.3. Assume that $X$ is an $F$-space and $T \in \mathcal{L}(X)$.

(a) If $T$ is topologically transitive, then, for any (open) neighbourhood $W$ of 0 , all sets $\mathbf{N}(V, W)$ and $\mathbf{N}\left(W, V^{\prime}\right)$ are thick.

(b) For $W$ neighbourhood of 0 and $V, V^{\prime}$ open, set $\mathbf{N}\left(V, W, V^{\prime}\right):=\mathbf{N}(V, W) \cap$ $\mathbf{N}\left(W, V^{\prime}\right)$. If all sets $\mathbf{N}\left(V, W, V^{\prime}\right)$ are nonempty, then all these sets are thick.

Proof. To prove (a), assume that $T$ is topologically transitive. Let us fix $V, W, V^{\prime}$ and some positive number $L$. Since $T(0)=0$, one can find an open neighbourhood $\widetilde{W}$ of 0 such that $T^{k}(\widetilde{W}) \subset W$ for all $k \in\{0 ; \ldots ; L\}$. Since $T$ is topologically transitive, 
one can find $n, n^{\prime} \in \mathbb{N}$ such that $T^{n}(V) \cap \widetilde{W} \neq \varnothing$ and $T^{n^{\prime}}(\widetilde{W}) \cap T^{-L}\left(V^{\prime}\right) \neq \varnothing$. If $k \in\{0 ; \ldots ; L\}$, then $n+k \in \mathbf{N}(V, \widetilde{W})+\mathbf{C}(\widetilde{W}, W) \subset \mathbf{N}(V, W)$, and $n^{\prime}+k=$ $\left(n^{\prime}+L\right)-(L-k) \in \mathbf{N}\left(\widetilde{W}, V^{\prime}\right)-\mathbf{C}(\widetilde{W}, W) \subset \mathbf{N}\left(W, V^{\prime}\right)$. Thus $\mathbf{N}(V, W)$ contains the interval $[n, n+L]$ and $\mathbf{N}\left(W, V^{\prime}\right)$ contains $\left[n^{\prime}, n^{\prime}+L\right]$. This proves (a). The proof of (b) is exactly the same: just note that in that case one may take $n=n^{\prime}$.

Part (b) of the above lemma immediately yields that if $X$ is an $F$-space, then a linear operator $T \in \mathcal{L}(X)$ is weakly mixing iff it satisfies the so-called three open sets condition:

(7) for any $V, V^{\prime} \subset X$ nonempty open and each $W$ neighbourhood of 0 , one can find $n \in \mathbb{N}$ such that $T^{n}(V) \cap W \neq \varnothing$ and $T^{n}(W) \cap V^{\prime} \neq \varnothing$.

The main point is that since $T$ is a linear map, we have

$$
\mathbf{N}(A, W) \cap \mathbf{N}\left(W, A^{\prime}\right) \subset \mathbf{N}\left(A+W, A^{\prime}+W\right),
$$

for any $A, A^{\prime}, W$. Since for any $V, V^{\prime}$, one can find nonempty open sets $A, A^{\prime}$ and a neighbourhood $W$ of 0 such that $A+W \subset V$ and $A^{\prime}+W \subset V^{\prime}$, it follows indeed at once from (b) above that (7) entails weak mixing.

Lemma 7.3 also gives the result of Grosse-Erdmann and Peris Proposition 5.5: since each set $\mathbf{N}(V, W)$ is thick, it intersects all $\mathbf{N}\left(W, V^{\prime}\right)$ if the latter are syndetic (which is the case under the assumption of Proposition 5.5), so that $T$ satisfies the three open sets condition (7).

It should be added that some authors call topologically ergodic a continuous map $T: X \rightarrow X$ for which all sets $\mathbf{N}\left(V, V^{\prime}\right)$ are syndetic (see [GlW]). Thus, Proposition 5.5 says that if a linear operator is topologically ergodic, then it is weakly mixing. This is false in the nonlinear setting.

7.4. Two examples. We now illustrate the usefulness of Theorem 7.2 by giving rather transparent proofs of two recent results in linear dynamics. The first one is due to S. Grivaux ([Gr]). In $[\mathrm{Gr}]$, it was the main step for showing that an operator $T \in \mathcal{L}(X)$ is weakly mixing if and only if the sequence $\left(T^{n_{k}}\right)$ is universal, for any syndetic sequence $\left(n_{k}\right) \subset \mathbb{N}$. The latter result was obtained independently by $\mathrm{A}$. Peris and L. Saldivia ([PS]) as a consequence of Theorem 7.2. Observe in particular that in condition (8) below, linearity is not needed.

Proposition 7.4. Weak mixing of $T$ is equivalent to the following property.

(8) For any $V, V^{\prime}$, one can find $k \in \mathbb{N}$ with $T^{k}(V) \cap V^{\prime} \neq \varnothing$ and $T^{k+1}(V) \cap V^{\prime} \neq \varnothing$. If $X$ is an $F$-space and $T \in \mathcal{L}(X)$, this is also equivalent to

(8') There exists some fixed positive integer $p$ such that: for any $V, V^{\prime}$, one can find $k \in \mathbb{N}$ with $T^{k}(V) \cap V^{\prime} \neq \varnothing$ and $T^{k+p}(V) \cap V^{\prime} \neq \varnothing$ 
Proof. Clearly, weak mixing implies (8). Conversely, assume that (8) holds; in other words, that

$$
1 \in \mathbf{N}\left(V, V^{\prime}\right)-\mathbf{N}\left(V, V^{\prime}\right)
$$

for any nonempty open sets $V, V^{\prime} \subset X$.

All sets $\mathbf{N}\left(V, V^{\prime}\right)$ are nonempty, hence $T$ is hypercyclic; let $x \in X$ be a hypercyclic point for $T$. Then

$$
\begin{aligned}
\mathbf{N}\left(V, V^{\prime}\right)-\mathbf{N}\left(V, V^{\prime}\right) & =\left[\mathbf{N}\left(x, V^{\prime}\right)-\mathbf{N}(x, V)\right]-\left[\mathbf{N}\left(x, V^{\prime}\right)-\mathbf{N}(x, V)\right] \\
& =\mathbf{N}\left(V^{\prime}, V^{\prime}\right)-\mathbf{N}(V, V),
\end{aligned}
$$

so that, for any $V, V^{\prime}$ we also have

$$
1 \in \mathbf{N}\left(V^{\prime}, V^{\prime}\right)-\mathbf{N}(V, V) .
$$

Now we show by induction on $m \in \mathbb{N}$ that property (5) in Theorem 7.2 is satisfied; that is, we show that for any $V, V^{\prime}$, one can find $k \in \mathbb{N}$ such that $T^{k}(V) \cap V^{\prime} \neq \varnothing$ and $T^{k+m}(V) \cap V^{\prime} \neq \varnothing$. The result holds for $m=0$ because $T$ is topologically transitive. Assume the result has been proved for $m$, and let us fix $V, V^{\prime}$. By induction hypothesis, one can find $k \in \mathbb{N}$ and two open sets $V_{1}, V_{2} \subset V$ such that $T^{k}\left(V_{1}\right) \subset V^{\prime}$ and $T^{k+m}\left(V_{2}\right) \subset V^{\prime}$. Then, by $(*)$, one can find $l \in \mathbb{N}$ such that $T^{l}\left(V_{1}\right) \cap V_{1} \neq \varnothing$ and $T^{l+1}\left(V_{2}\right) \cap V_{2} \neq \varnothing$. Thus, we get $l+k \in \mathbf{N}\left(V_{1}, V_{1}\right)+\mathbf{C}\left(V_{1}, V^{\prime}\right) \subset \mathbf{N}\left(V, V^{\prime}\right)$, and likewise $(l+k)+(m+1)=(l+1)+(k+m) \in \mathbf{N}\left(V, V^{\prime}\right)$, which proves (5) for $m+1$. This concludes the proof for $(8)$.

Now, assume that ( $\left.8^{\prime}\right)$ holds in the linear setting, for some positive integer $p$. Then $T$ is still hypercyclic, whence $T^{p}$ is also hypercyclic, by Ansari's Theorem ([A]). One can then show exactly as above that (5) holds for $T^{p}$. Thus, $T^{p}$ is weakly mixing, hence so is $T$.

Our second illustration is a result due to G. Costakis and M. Sambarino ([CS]). Recall that a continuous map $T$ is said to be mixing if all sets $\mathbf{N}\left(V, V^{\prime}\right)$ are cofinite. This should be compared with $7.2(2)$, which yields by a simple diagonal argument that weak mixing is equivalent to the following property: each set $\mathbf{N}\left(V, V^{\prime}\right)$ contains a cofinite subset of some fixed infinite set $\mathbf{N} \subset \mathbb{N}$. In case $T$ is linear and satisfies the hypercyclicity criterion with respect to some sequence $\left(n_{k}\right)$, one can take $\mathbf{N}=$ $\left\{n_{k} ; k \in \mathbb{N}\right\}$.

Proposition 7.5. Assume that $X$ is an $F$-space and that $T \in \mathcal{L}(X)$. If $T$ satisfies the Hypercyclicity Criterion with respect to some syndetic sequence $\left(n_{k}\right)$, then $T$ is mixing.

Proof. Set $\mathbf{N}:=\left\{n_{k} ; k \in \mathbb{N}\right\}$, and let us denote by $\mathcal{F}$ the family of all subsets of $\mathbb{N}$ containing some cofinite subset of $\mathbf{N}$. Then $\mathcal{F}$ is a filter of subsets of $\mathbb{N}$, and by assumption we have $\mathbf{N}\left(V, V^{\prime}\right) \in \mathcal{F}$ for any $V, V^{\prime}$. Moreover, there exists some fixed $K>0$ such that the following holds: for each each set $\mathbf{F} \in \mathcal{F}$, one can find $a \in \mathbb{N}$ 
such that $\mathbf{F}$ meets every interval $I$ of length $K$ with $\min (I) \geq a$. In short, we shall say that the sets in $\mathcal{F}$ are $K$-syndetic.

Now, let us fix $V, V^{\prime}$. For each $k \in\{0 ; \ldots ; K\}$, one can find some nonempty open set $V_{k}$ such that $T^{k}\left(V_{k}\right) \subset V^{\prime}$. Then $\mathbf{F}:=\bigcap_{k=0}^{K} \mathbf{N}\left(V, V_{k}\right) \in \mathcal{F}$ because $\mathcal{F}$ is a filter. Therefore, $\mathbf{F}$ is $K$-syndetic, so that $\bigcup_{k=0}^{K}(\mathbf{F}+k)$ is a cofinite subset of $\mathbb{N}$. But

$$
\bigcup_{k=0}^{K}(\mathbf{F}+k) \subset \bigcup_{k=0}^{K}\left(\mathbf{N}\left(V, V_{k}\right)+\mathbf{C}\left(V_{k}, V^{\prime}\right)\right) \subset \mathbf{N}\left(V, V^{\prime}\right),
$$

hence $\mathbf{N}\left(V, V^{\prime}\right)$ is cofinite as well.

Remark. What was really used in the above proof is the following fact: one can find some filter $\mathcal{F}$ such that $\mathbf{N}\left(V, V^{\prime}\right) \in \mathcal{F}$ for any $V, V^{\prime}$, and each set $\mathbf{F} \in \mathcal{F}$ is $K$-syndetic. Once this is known, linearity is not needed.

\section{REFERENCES}

[A] S. Ansari, Hypercyclic and cyclic vectors. J. Funct. Anal. 128 (1995), no. 2, 374-383.

[BaGr] F. Bayart, S. Grivaux, Frequently hypercyclic operators. Trans. Amer. Math. Soc. 358 (2006), no. 11, 5083-5117.

[BaM] F. Bayart, E. Matheron, Hypercyclic operators failing the Hypercyclicity Criterion on classical Banach spaces. J. Funct. Anal. 250 (2007), 426-441.

[BeP] J. Bès, A. Peris, Hereditarily hypercyclic operators. J. Funct. Anal. 167 (1999), no. 1, 94-112.

[BonGE] A.Bonilla, K. -G. Grosse-Erdmann, Frequently hypercyclic operators and vectors. Ergodic Theory Dynam. Systems 27 (2007), 383-404.

[BouF] P. S. Bourdon, N. S. Feldman, Somewhere dense orbits are everywhere dense. Indiana Univ. Math. J. 52 (2003), no. 3, 811-819.

[CS] G. Costakis, M. Sambarino, Topologically mixing hypercyclic operators. Proc. Amer. Math. Soc. 132 (2004), no. 2, 385-389.

[DR] M. De La Rosa, C. J. Read, A hypercyclic operator whose direct sum is not hypercyclic. Journal of Operator Theory (to appear).

[F] H. Furstenberg, Recurrence in ergodic theory and combinatorial number theory. Princeton University Press (1981).

[Gl] E. Glasner, Ergodic theory via joinings. Mathematical Surveys and Monographs 101, American Mathematical Society (2003).

[GlW] E. Glasner, B. Weiss, On the interplay between mesurable and topological dynamics. Handbook of dynamical systems, vol. 1B, 597-648, Elsevier B. V. (2006).

[Gr] S. Grivaux, Hypercyclic operators, mixing operators, and the bounded steps problem. J. Operator Theory 54 (2005), no. 1, 147-168.

[GEP] K. -G. Grosse-Erdmann, A. Peris, Frequently dense orbits. C. R. Acad. Sci. Paris 341 (2005), 123-128.

[Hal] H. Halberstam, K. F. Roth, Sequences. Springer-Verlag (1983).

[PS] A. Peris, L. Saldivia, Syndetically hypercyclic operators. Integral Equations Operator Theory 51 (2005), no. 2, 275-281.

[Rus] I. Z. Rusza, An infinite Sidon sequence. J. Number Theory 68 (1998), 63-71. 
Laboratoire de Mathématiques, Université Blaise Pascal, Campus universitaire des CÉzeaux, 63177 Aubières Cedex

E-mail address: Frederic.Bayart@math.univ-bpclermont.fr

Laboratoire de Mathématiques de Lens, Université D’Artois, Rue Jean Souvraz S. P. 18, 62307 LENS

E-mail address: etienne.matheron@euler.univ-artois.fr 